\title{
1 Antibody feedback limits the expansion of cognate memory B cells but drives the
}

4 Hayley A. McNamara ${ }^{1}$, Azza H. Idris ${ }^{2}$, Henry J. Sutton ${ }^{1}$, Barbara J. Flynn ${ }^{2}$, Yeping Cai ${ }^{1}$, Kevin

5 Wiehe $^{3,4}$, Kirsten E. Lyke ${ }^{5}$, Deepyan Chatterjee ${ }^{1}$, Natasha KC ${ }^{6}$, Sumana Chakravarty ${ }^{6}$, B. Kim

6 Lee $\operatorname{Sim}^{6}$, Stephen L. Hoffman ${ }^{5}$, Mattia Bonsignori ${ }^{3,4}$, Robert A. Seder ${ }^{2}$ and Ian A. Cockburn ${ }^{1 *}$

8 1. Department of Immunology and Infectious Disease, John Curtin School of Medical Research,

9 The Australian National University, Canberra, ACT, 2601, Australia

10 2. Vaccine Research Center (VRC), National Institute of Allergy and Infectious Diseases,

11 National Institutes of Health, Bethesda, MD 20892, USA

12 3. Duke Human Vaccine Institute, Durham, NC 27710, USA

13 4. Department of Medicine, Duke University Medical Center, Durham, NC 27710, USA

14 5. Center for Vaccine Development and Global Health, University of Maryland School of

15 Medicine, Baltimore, MD 21201, USA

16 6. Sanaria Inc., Rockville, MD 20850, USA

$18 *$ Corresponding author

20 email: ian.cockburn@anu.edu.au

21 tel: +61261254619 


\section{Abstract}

24 Generating sufficient antibody to block infection is a key challenge for vaccines against malaria.

25 Here we show that antibody titres to a key target, the repeat region of the Plasmodium

26 falciparum circumsporozoite protein (PfCSP), plateaued after two immunizations in a clinical

27 trial of the radiation-attenuated sporozoite vaccine. To understand the mechanisms limiting

28 vaccine responsiveness, we developed Ig-knockin mice with elevated numbers of PfCSP-binding

29 B cells. We determined that recall responses were inhibited by antibody feedback via epitope

30 masking of the immunodominant PfCSP repeat region. Importantly, the amount of antibody that

31 prevents boosting is below the amount of antibody required for protection. Finally, while

32 antibody feedback limited responses to the PfCSP-repeat region in vaccinated volunteers,

33 potentially protective subdominant responses to C-terminal regions did expand with subsequent

34 boosts. These data suggest that antibody feedback drives the diversification of immune responses

35 and that vaccination for malaria will require the targeting of multiple antigens. 
Introduction

39 Induction and maintenance of humoral immunity is the mechanism of protection for most

40 licenced vaccines against viral or bacterial infections. Most of these effective vaccines target

41 antigenically simple infections which induce robust immune memory once the infections resolve.

42 Even allowing for different methods to quantitate antibody titer or function, protection by these

43 vaccines can often be mediated by relatively low amounts of specific antibodies $(\sim 0.1-10 \mu \mathrm{g})^{1}$.

44 The low amounts of antibody required for neutralization, coupled with the fact that antibody

45 responses can have very long half-lives (10-300 years) $)^{2}$ allows our most effective vaccines to

46 confer life-long immunity.

48 In contrast to current successful vaccine approaches, high antibody titers are likely to be required 49 to protect against complex pathogens such as Plasmodium falciparum and HIV ${ }^{3,4,5}$. For malaria 50 the most advanced vaccine, RTS,S, targets the $P$. falciparum circumsporozoite protein (PfCSP)

51 which coats the surface of the Plasmodium sporozoite. RTS,S given with the very potent AS01B

52 adjuvant is administered three times at 4 week intervals and induces very high levels of

53 antibodies $(>100 \mu \mathrm{g} / \mathrm{ml})$ against the immunodominant $(\mathrm{NANP})_{\mathrm{n}}$ repeat region within PfCSP;

54 however these titers wane rapidly and this is associated with diminished protection over time ${ }^{4,5}$,

$55{ }^{6}$. Anti-(NANP) $)_{\mathrm{n}}$ repeat responses saturate after 2 immunizations and a booster at 18 months

56 provides only a modest increase in antibody and protection ${ }^{4,5,6,7}$.

58 An alternative vaccine approach has been to develop an attenuated whole parasite vaccine using radiation attenuated $P$. falciparum sporozoites $(\mathrm{PfSPZ})^{8}$. This vaccine confers sterile protection 
60 in malaria-naïve individuals for $\sim 1$ year which is thought to be mediated largely by $\mathrm{T}$ cells in the

61 liver ${ }^{8,9,10,11}$. However, there is also evidence that PfSPZ Vaccine-induced antibodies may have

62 some short-term protective role and utility as a correlate of protection ${ }^{8,10}$.

63

64 Given the limited capacity of these current malaria vaccine approaches to induce sustained

65 antibody mediated protection, it is critical to determine the mechanisms underlying B cell

66 responses to Plasmodium sporozoites and PfCSP in particular ${ }^{12,13,14,15}$. Analysis of antibody

67 titer, breadth and single cell antigen specific B cell responses to RTS,S and PfSPZ vaccines in

68 humans provides critical hypothesis generating data for developing mouse models to establish

69 mechanisms ${ }^{8,10,16,17,18}$. Here we show that following immunization with PfSPZ Vaccine in

70 humans, B cells lose responsiveness after 2 vaccinations. To dissect the mechanism of this non-

71 responsiveness in vivo, we developed Ig-knockin mice specific for PfCSP that facilitate tracking

72 of the B cell responses to PfCSP. The data reported herein show that the lack of B cell boosting

73 was mediated by antibody feedback by repeat-specific antibodies. However, boosting led to the

74 emergence of subdominant epitopes and increased the diversity of the antibody response over

75 time. This suggests that effective vaccination may depend on inducing responses to a diverse

76 range of protective epitopes. 


\section{$77 \quad$ Results}

Memory B cells specific for PfCSP show limited recall after 2 vaccinations

81 To first determine the humoral response after sequential vaccination in humans, we examined

82 antibody responses against PfCSP in U.S. malaria-naïve adults who received 3 doses of $9 \times 10^{5}$

83 PfSPZ Vaccine each at 8-week intervals as part of a clinical trial of this whole parasite vaccine

84 (Figure $1 \mathrm{~A})^{11}$. The total anti-PfCSP antibody response increased significantly after the primary

85 (V1) vaccination and second (V2) vaccination but did not increase following an additional boost

86 (V3) (Figure 1B). We hypothesised that this lack of boosting could be attributed to a reduced

87 efficiency in engaging memory B cells as part of a recall response. To investigate this, 1 week

88 after each vaccination individual plasmablasts (PBs) from 3 randomly selected individuals were

89 sorted and their rearranged Ig V(D)J genes amplified and cloned. Monoclonal antibodies were

90 expressed from these rearranged $\mathrm{Ig} \mathrm{V}(\mathrm{D}) \mathrm{J}$ sequences and screened for reactivity against PfCSP

91 19, 20,21. After V1, only 13/153 (8.5\%) PBs isolated from these 3 individuals were PfCSP-specific

92 (Supplementary Dataset; Figure 1C). However, after V2, 45/138 (32.6\%) PBs, were specific for

93 PfCSP, many of these (29/45) used the IGHV3-33 gene, and 18/45 belonged to one of 7

94 expanded clones, indicative of a robust B cell memory response at this timepoint (Figure 1C).

95 After V3 however, only 16/112 (14.2\%) PBs were PfCSP specific, suggesting that the memory B

96 cell recall response is diminished after the first boost, consistent with the lack of increase in

97 antibody titers at this timepoint (Figure 1C). 
99 Analysis of the antibody sequences revealed that the level of somatic hypermutation in PfCSP

100 specific antibodies was comparable to non-PfCSP binding antibodies at V1, but much lower at

101 V2 (Figure 1D). This is consistent with the initial PB response at V1 coming from pre-existing

102 cross-reactive memory cells as has been proposed previously ${ }^{15}$ while PBs recruited at V2

103 possibly come from memory cells that were originally primed at V1. Also in agreement with

104 previous data ${ }^{15}$, we find that $8 / 25$ PfCSP binding clones that use the $I G H V 3-33$ paired with a

105 light chain formed from the IGKV1-5/IGKJI in which there were no n-nucleotide insertions

106 between the $I G K V$ and $I G K J$ gene segments, resulting in a public IgL. Despite the evidence for

107 pre-formed high affinity antibodies in multiple individuals and the low levels of SHM, many of

108 these antibodies do appear to have undergone affinity maturation. In particular 17/25 PfCSP-

109 specific clones that use the IGHV3-33 gene carry mutations at either position 107 or 163 (Figure

110 1E; Supplementary Dataset). These mutations in the IGHV3-33 gene commonly encode for

111 S32N and V55I mutations at the protein level (Figure 1F). Structural analysis of a potent repeat

112 binding antibody (mAb311) that carries both these mutations in the IGHV3-33 heavy chain has

113 shown that these residues are important for binding to PfCSP ${ }^{22}$ further suggesting that these

114 antibodies are under selection. Collectively, our data are consistent with specific PfCSP-binding

115 memory cells being initially primed at V1, and then responding robustly in a secondary response

116 to PfSPZ; however, on subsequent boosting these cells become eliminated, exhausted or limited

117 in their expansion.

119 A mouse model to investigate the PfCSP-specific B cell responses to sporozoites 
121 To gain insight into the mechanism of the limited B cell boosting following irradiated sporozoite

122 vaccination, we developed Ig-knockin mice - designated $\operatorname{Igh}^{\mathrm{g} 2 \mathrm{~A} 10}-$ in which the germline-

123 reverted (unmutated) heavy chain VDJ exon (Ighv9-3, Ighd1-3, Ighj4) from 2A10, a murine

124 PfCSP-neutralising antibody ${ }^{13,23,24}$, was inserted upstream of the IgM locus (Supplementary

125 Figure $2 \mathrm{~A}$ ). Within these mice $\sim 2 \%$ of the $\mathrm{B}$ cell repertoire is specific for the PfCSP repeat

126 region compared with $\sim 0.04 \%$ of B cells within C57BL/6 controls (Figure $2 \mathrm{~A}$ ), the fact that only

$127 \sim 2 \%$ of B cells in Igh ${ }^{\mathrm{g} 2 \mathrm{~A} 10}$ mice are PfCSP specific is presumably due to the fact that the inserted

$128 I g h^{\mathrm{g} 2 \mathrm{~A} 10}$ heavy chain remains free to pair with any endogenous light chain. Moreover, because the

$129 \operatorname{Ig} h^{\mathrm{g} 2 \mathrm{~A} 10}$ gene was inserted upstream of the $\operatorname{IgM}$ locus these antigen specific cells in naïve mice

130 were either $\operatorname{IgD}^{+}$or $\operatorname{IgM}^{+}$(Figure $2 \mathrm{~A}$ ). The affinity of these cells for PfCSP was estimated by

131 preincubation of $\operatorname{Igh}^{\mathrm{g} 2 \mathrm{~A} 10}$ splenocytes with titrated amounts of PfCSP prior to tetramer staining

132 (Figure 2B). Based on the $\mathrm{EC}_{50}$ of the inhibition of tetramer staining we estimated the affinity of

133 Igh ${ }^{\mathrm{g} 2 \mathrm{~A} 10}$ cells for PfCSP to be $\sim 1.33 \times 10^{-7} \mathrm{M}$ which is $\sim 50$-fold lower that the affinity of the $2 \mathrm{~A} 10$

$134 \mathrm{mAb}$ for PfCSP $\left(2.7 \times 10^{-9} \mathrm{M}\right)^{13}$ consistent with the insertion of the unmutated germline VDJ

135 heavy chain sequence rather than the mutated VDJ of the mature $2 \mathrm{~A} 10 \mathrm{mAb}$. Overall the

136 development of total B cells was largely normal in $\operatorname{Igh}^{\mathrm{g} 2 \mathrm{~A} 10}$ mice with all spleen, bone marrow,

137 lymph node and blood B cell populations present, though we did note a reduced number of B1a

138 B cells and a bias towards the formation of marginal zone B cells compared to follicular B cells,

139 perhaps because of the restricted B cell receptor (BCR) repertoire of these mice (Supplementary

140 Figure 2B-F) ${ }^{25,26}$.

142 To test whether the PfCSP-specific B cells from $\operatorname{Igh}^{\mathrm{g} 2 \mathrm{~A} 10}$ mice respond to antigen, $1 \times 10^{4}$

143 congenically marked CD $45.1^{+}$tetramer $^{+} \operatorname{Igh}^{\mathrm{g} 2 \mathrm{~A} 10} \mathrm{~B}$ cells were adoptively transferred into naïve 
144 CD45.2 $2^{+}$C57BL/6 recipient mice, which were then vaccinated IV with radiation attenuated $P$.

145 berghei parasites that have been engineered to express PfCSP ${ }^{27}$ (Pb-PfCSP SPZ); control mice

146 received salivary gland extract (SGE) from uninfected Anopheles mosquitoes as sporozoites have

147 to be dissected from infected mosquitoes (Figure 2C). Pb-PfCSP SPZ infected mice developed

148 early IgM responses that waned rapidly, and IgG responses that developed strongly from day 7

149 and reached a peak at day $25-28$ of $\sim 20 \mu \mathrm{g} / \mathrm{ml}$. No significant anti-PfCSP response was observed

150 in mice immunized with salivary gland extract alone (Figure 2D). Following immunization with

$1515 \times 10^{4} \mathrm{~Pb}$-PfCSP SPZ, flow cytometry analysis (Supplementary Figure $2 \mathrm{G}$ ) of the Igh ${ }^{\mathrm{g} 2 \mathrm{~A} 10}$ cells

152 in the spleen revealed extensive expansion and class switching of the B cells after 4 days (Figure

$1532 \mathrm{E}$ and $\mathrm{F})$. The early response is dominated by PBs, which subsides as germinal center (GC) and

154 memory B cells increase, and peak, after two weeks (Figure 2E and G). Interestingly, GC B cells

155 appear to persist for an extended period $>60$ days, when compared to commonly used

156 immunisation models including HEL-SRBC and NP conjugates in which GCs resolve after $\sim 1$

157 month ${ }^{28,29}$.

159 Because sustained antibody responses depend on the formation of a pool of long-lived bone

160 marrow plasma cells (BMPCs) ${ }^{30,31}$, we further developed a system to facilitate the identification

161 of these cells. Accordingly, CD45.1 $\mathrm{Igh}^{\mathrm{g} 2 \mathrm{~A} 10}$ mice were crossed to a Blimp ${ }^{\mathrm{GFP} /+}$ reporter mouse;

162 Blimp1 is a key transcription factor for maintaining the plasmacell program and these mice

163 express high levels of GFP in long-lived $\mathrm{BMPCs}^{32}$. In these mice, we were able to identify a

164 population within the bone marrow, which were GFPhi $\mathrm{CD}^{2} 5.1^{+}$and $\mathrm{CD} 138^{\mathrm{hi}}$ cells

165 approximately half of which bound our (NANP) 9 -tetramers (Supplementary Figure 2H; Figure

$1662 \mathrm{H}$ ). Accordingly, we sorted tetramer ${ }^{+}$and tetramer- $\mathrm{CD}^{-} 5.1^{+} \mathrm{GFP}^{+}$cells from the bone marrow 
167 onto PfCSP coated ELISpot plates and determined that only the tetramer ${ }^{+}$cells secreted PfCSP

168 specific antibody, confirming these as antibody-secreting BMPCs (Figure 2H and I).

170 Finally to determine if efficient somatic hypermutation occurs in our Igh ${ }^{\mathrm{g} 2 \mathrm{~A} 10}$ cells, tetramer $^{+}$

171 BMPCs were sorted and the recombined Ig V(D)J heavy and light chains sequenced using single

172 cell RNA-seq ${ }^{33}$. Single cell RNA-seq was used as we did not know the identity of the light

173 chains in our PfCSP binding cells. Blimp ${ }^{\mathrm{GFP} /+}, \mathrm{CD} 45.1^{+}, \operatorname{Igh}^{\mathrm{g} 2 \mathrm{~A} 10}$ cells exclusively used the

$174 \operatorname{Ighv9-3}$ heavy chain allele paired with the Igkv10-94 light chain allele which is also used by the

175 2A10 antibody. Interestingly RNA-seq data revealed that the BMPCs were a mix of $\operatorname{IgM}^{+}$and

$176 \mathrm{IgG}^{+}$cells. While $\sim 75 \%$ of $\mathrm{IgM}^{+}$cells were unmutated, $\sim 90 \%$ of $\mathrm{IgG}^{+} \mathrm{BMPCs}$ were somatically

177 mutated, with all mutated cells carrying the L114F mutation in the kappa chain which has

178 previously been shown to drive affinity maturation in the 2A10 mAb (Supplementary Figure 3 ).

179 The acquisition of a low number of critical mutations indicates that affinity maturation is taking

180 place in these B cells in a similar manner to PfCSP specific B cells in humans (Figure 1E-F).

181 Overall the data shows that the Igh ${ }^{\mathrm{g} 2 \mathrm{~A} 10}$ knockin cells form PBs, GC B cells, memory cells and

182 BMPCs similar to endogenous cells. Moreover, they undergo class switching and affinity

183 maturation in a physiological manner that appears to replicate the B cell differentiation observed

184 in PfSPZ-vaccinated humans.

186 PfCSP-specific antibody and B cell responses have limited boosting in a murine model

188 To investigate the mechanism underlying the limited recall responses of PfCSP-specific B cells 189 in human vaccinees following PfSPZ Vaccine, $2 \times 10^{4}$ Blimp $^{\mathrm{GFP} /+}, \mathrm{CD} 45.1^{+}, \mathrm{Igh}^{\mathrm{g} 2 \mathrm{~A} 10} \mathrm{~B}$ cells were 
190 transferred into congenic recipients, which were vaccinated with $5 \times 10^{4}$ irradiated $\mathrm{Pb}-\mathrm{PfCSP}$

191 SPZ (denoted V1, V2, V3 analogously to the clinical trials in humans) at one-month intervals

192 between each dose (Figure 3A). Additional control groups of mice received either just one (V1)

193 or two immunizations (V1+V2). Anti-PfCSP titers peaked after V2 at $\sim 70 \mu \mathrm{g} / \mathrm{ml}$ before

194 declining to $\sim 40 \mu \mathrm{g} / \mathrm{ml}$ and, similar to vaccinated humans, this response was not enhanced by a

195 further boost (Figure 3B). We further analysed the PB, GC and memory cell responses at the

196 cellular level by flow cytometry (Figure 3C and D). We detected a transient expansion of the PB

$197\left(\mathrm{CD} 45.1^{+}, \mathrm{CD} 138^{\mathrm{hi}}, \mathrm{GFP}^{\mathrm{lo}}\right)$ and memory $\left(\mathrm{CD} 45.1^{+} \mathrm{CD} 38^{+}\right)$response after V2 but not V3 (Figure

$1983 \mathrm{E}$ and $\mathrm{F}$ ); however, there was no change in the ongoing $\mathrm{GC}$ response (Figure 3G). Boosting was

199 associated with a small increase in BMPCs after V2 that was marginally significant, however a

200 third boost did not enhance numbers further (Figure 3H). Taken together, these data are

201 consistent with the observations in humans (Figure 1B and C) that memory cells can be recalled

202 into the response at V2, but not at V3 limiting the titer of neutralizing antibody that can be

203 achieved.

204

205 Sporozoite-induced memory cells are functional

207 The next series of studies determined why the recall response was so rapidly diminished. One

208 hypothesis is that Pb-PfCSP SPZ immunization induces non-responsive memory B cells. To

209 directly test this, memory cell populations were generated by transferring $\operatorname{Igh}^{\mathrm{g} 2 \mathrm{~A} 10} \mathrm{~B}$ cells to

210 congenic mice and immunizing with either Pb-PfCSP SPZ or recombinant PfCSP (rPfCSP) in

211 alum as a proxy for a recombinant protein immunization. After $>2$ months, switched memory B

212 cells which expressed PD-L2 and CD80, markers of functional memory B cells were detected ${ }^{34}$, 
213 suggesting normal differentiation (Supplementary Figure 4A and B). Negative selection was then

214 used to enrich antigen experienced memory B cells from immunized mice and remove

215 contaminating plasma cells and GC B cells (Supplementary Figure 4C). The functional capacity

216 of the memory B cells was assessed by adoptive transfer into naïve mice and re-immunization

217 with $5 \times 10^{4} \mathrm{~Pb}$-PfCSP SPZ (Figure 4A). Immunized mice that had received memory cells had

218 significantly higher titers of PfCSP-specific IgG, but not IgM, than immunized mice that did not

219 receive cells (Figure 4B), indicating that the transferred memory cells could differentiate into

220 antibody secreting cells upon recall. At the cellular level, memory cells from Pb-PfCSP SPZ

221 immunized mice expanded $\sim 50$ fold in 5 days, and differentiated into both CD138 $8^{+}$PBs and

$222 \mathrm{GL7}^{+} \mathrm{GC}$ B cell precursors (Figure 4C-E). This level of expansion was only slightly lower that

223 that seen among memory B cells primed with rPfCSP in Alum. Overall, our experiments indicate

224 that irradiated sporozoite immunization induces memory cells that appear capable of mounting

225 robust recall responses.

PfCSP specific memory B cells are inhibited by antibody feedback

229 Given that the PfCSP-specific memory B cells were functional in naïve mice, we next

230 investigated whether they were regulated by other components of the ongoing immune response.

231 Accordingly, memory B cells were adoptively transferred 1 month after immunization into

232 immune-matched mice that had received a Pb-PfCSP SPZ immunization (Figure 5A). In

233 immune-matched mice, the expansion of transferred memory B cells was significantly limited,

234 with only a small number of cells differentiating into PBs (Figure 5B-C). This regulation did not

235 appear to be mediated by the cellular response as Pb-PfCSP SPZ pre-immune MD4 mice, which 
236 have an Ig specific for hen egg lysozyme and should not produce PfCSP-specific antibody ${ }^{35}$ but

237 have normal $\mathrm{T}$ cell responses, failed to show the same inhibition as the pre-immune C57BL/6

238 mice. Further, passive transfer of immune sera was sufficient to severely limit the memory B cell

239 response (Figure 5B-C). Overall the inhibition of memory B cell expansion appeared to correlate

240 with the level of anti-(NANP) $\mathrm{n}$ antibodies (Figure 5D). If antibody feedback was the mechanism

241 limiting memory responses in human PfSPZ vaccinees, there should be an inverse relationship

242 between the amount of antibody prior to a booster immunization and the subsequent change in

243 the antibody titer. In agreement with this, there was a strong inverse correlation between post V2

244 antibody titres and V3 boosting $\left(r^{2}=0.51 ; p=0.0041\right)$ in vaccinated humans, indicative of

245 strong antibody feedback regulation limiting the third immunization (Figure 5E). Conversely, no

246 such relationship was observed at the earlier V2 immunization $\left(r^{2}=0.12 ; p=0.22\right)$, indicating

247 that antibody feedback was not strongly regulating B cell responses at this earlier timepoint.

249 We further predicted, that if antibody feedback regulates recall responses, then delaying boosting

250 until antibody titers have declined would enhance recall responses. Accordingly, in two groups

251 of mice we delayed the first boost until 6 months after the initial priming immunization

252 (Supplementary Figure 5A). In these mice titres peaked at $\sim 100 \mu \mathrm{g} / \mathrm{ml}$ (Supplementary Figure

$2535 \mathrm{~B})$, which was higher than the peak in mice that received a boost at $1 \mathrm{month}(\sim 70 \mu \mathrm{g} / \mathrm{ml}$; Figure

254 3B). The magnitude of the secondary PB response was not obviously larger in these mice than in

255 mice that received a boost one month after the prime (Supplementary Figure 5C-D), but the

256 delayed mice exhibited secondary GCs and so the B cells may have enhanced SHM and affinity

257 maturation resulting in higher titers of protective antibody (Supplementary Figure 5E-G). 
Sub-protective levels of anti-repeat antibodies block recall responses by memory B cells

261 For vaccination a critical metric is the protective threshold of antibody required for protection. If

262 this protective threshold is above the amount of antibody required to inhibit memory B cell

263 responses it will be more difficult to achieve sustained protective titers by vaccination.

264 Accordingly, we determined the protective threshold for the 2 A10 antibody by passively

265 transferring different amounts of antibody to mice and subsequently challenging them via bites

266 of Pb-PfCSP infected mosquitoes and following mice for 14 days or until they became infected

267 ( $>0.1 \%$ parasitaemia). A dose of $300 \mu \mathrm{g}$ provided complete protection in $7 / 10$ mice, but a dose of

$268100 \mu \mathrm{g}$ conferring only partial protection (3/10 mice protected; Figure 6A). Passive transfer of

$269100 \mu \mathrm{g}$ antibody resulted in a serum concentration of $\sim 70 \mu \mathrm{g} / \mathrm{ml}$ (Figure $6 \mathrm{~B}$ ) which is

270 approximately double the amount achieved by vaccination in our mice (Figure 3B) and similar to

271 the level of antibody required for $50 \%$ protection following RTS,S vaccination ${ }^{4}$. We next

272 determined the dose of antibody required to inhibit recall responses in mice by transferring

273 memory B cells to mice and subsequently boosting with Pb-PfCSP SPZ in the presence or

274 absence of antibody. 2A10 antibody inhibited B cell responses at all concentrations tested

275 (Figure 6C), with the lowest concentration of $33 \mu \mathrm{g}$ permitting only minimal differentiation of

276 CSP specific B cells (Figure 6D). These data are consistent with the fact that there was no

277 response at $\mathrm{V} 3$ in the mice at a timepoint when the serum titer of antibody was $\sim 70 \mu \mathrm{g} / \mathrm{ml}$

278 (Figure 3C).

280 To determine whether this effect is generalizable to other anti-PfCSP antibodies the ability of the

281 potent human neutralizing antibody CIS43 to inhibit the expansion of Ighg2A10 was 
282 investigated. CIS43 has dual binding activity with affinity for both the (NANP) $n$ repeat and the

283 junction between $\mathrm{R} 1$ and the repeat ${ }^{20}$. In broad agreement with our previous work we found that

284 concentrations of $\sim 15 \mu \mathrm{g} / \mathrm{ml}$ blocked around $50 \%$ of infections, significantly better than $2 \mathrm{~A} 10$

285 (Figure 6E-F). These serum concentrations also potently inhibited $\operatorname{Igh}^{\mathrm{g} 2 \mathrm{~A} 10}$ memory B cell

286 expansion, upon boosting, compared to mice that received no antibody, or control antibodies

287 with irrelevant specificities but not to the same degree as 2A10 (Figure 6G). Moreover, some of

288 the memory B cells that did expand were able to differentiation into PBs in the presence of semi-

289 protective levels of CIS43 (Figure 6H).

291 Antibody feedback occurs via epitope masking permitting the expansion of subdominant

292 responses

293

294 The final experiments were aimed at determining the mechanism of feedback inhibition by

295 antibody. In particular, whether antibody inhibition regulated PfSPZ specific B cell responses

296 generally or if the inhibition was antigen specific. General mechanisms of antibody feedback

297 would include action via inhibitory $\mathrm{Fc}$ receptors ${ }^{36}$, or clearance of parasite before parasite

298 antigens could enter presentation pathways ${ }^{37}$. Alternatively, if the antibodies were acting via

299 epitope masking the feedback would be epitope specific such that antibodies specific for other

300 regions of the CSP molecule would not inhibit responses by our (NANP) -repeat specific cells

301 (Figure 7A).

302

303 To determine if inhibitory Fc receptors were important, 2A10 LALA-PG mutant antibodies

304 carrying Leucine-to-Alanine mutations in positions 234 and 235, and a Proline-to-Glycine 
305 substitution at position 329 in the Fc portion of the antibody were made that limit binding with

306 Fc-gammaRIIB receptors ${ }^{38}$. These antibodies, however, inhibited the expansion of memory cells

307 similarly to the unmutated antibodies (Figure 7B). To determine if antibodies cleared parasite

308 and prevented parasite antigens being presented to B cells, we took advantage of the fact that

309 sporozoites rapidly infect the liver ( $<2$ hours even after intradermal immunization), and are

310 rapidly taken up into antigen presentation pathways in the spleen or draining lymph nodes ${ }^{39,40,}$

311 this should therefore be circumvented if the antibody was delivered late. However, transfer of the

312 antibody 4 hours after immunization only permitted a minimal expansion of memory B cells

313 (Figure 7B). Therefore, these two general mechanisms cannot explain the observed negative

314 antibody feedback. In contrast mAbs targeting either the N-terminal (5D5) ${ }^{41}$ or C-terminal

$315(\mathrm{mAb} 15)^{20}$ domains of PfCSP had little or no inhibitory effect on responses by memory Igh ${ }^{\mathrm{g} 2 \mathrm{~A} 10}$

316 cells (Figure 7B) which is consistent with epitope masking being the mechanism of antibody

317 feedback. This was true even when the 5D5 and mAb15 antibodies were added in significant

318 excess (Figure 7C). Importantly, dissociation constants for the binding of 5D5, mAb15 and 2A10

319 to PfCSP are all in the same range (Supplementary Figure 6).

321 If epitope masking was the mechanism of antibody feedback in humans that received PfSPZ

322 vaccine, then it should still be possible for responses to subdominant epitopes targeting other

323 regions of PfCSP to continue to expand even if the immunodominant anti-repeat response has

324 plateaued. We therefore separated responses in our vaccine cohort by specificity for the (NANP)n

325 repeat which is immune-dominant or the $\mathrm{C}$ terminus. As expected, responses to the (NANP)

326 repeat were similar to those of whole PfCSP, plateauing after V2. Of note, however C terminal

327 responses continued to increase after V3, suggesting antibody feedback was not acting on C- 
328 terminal responses (Figure 7D). In agreement with this there was correlation between V2 anti-

$329(\mathrm{NANP})_{\mathrm{n}}$ repeat titer and boost at V3 that was even stronger than that observed for total CSP

330 responses $\left(r^{2}=0.74, p<0.0001\right.$; Figure 7E). In contrast no relationship was observed between

331 antibody titer and the magnitude of boosting for C-terminal antibodies at any timepoint (Figure

332 7F). It is notable that C-terminal antibodies have been associated with protection by the RTS, S

333 vaccine ${ }^{42,43}$. Overall these data strongly support the hypothesis that epitope masking rather than

334 parasite clearance of Fc mediated immune regulator mechanisms account for antibody feedback

335 not only in the mouse model but also in vaccinated individuals. 


\section{Discussion}

The standard approach for generating high titers of antibody has been a series of immunizations

followed by periodic booster injections depending on the infection. For prevention of malaria it has proven difficult to achieve the necessary high titers to achieve high-level $(>50 \%)$ protective antibody even with additional boosting. Using data from a human clinical trial combined with novel Ig-knockin mouse model we show that boosting is limited by antibody feedback which

343 prevents antibodies to a single epitope reaching protective levels. While antibody feedback is a well-established immunological phenomenon $44,45,46$, its role in limiting vaccine responses to complex pathogens has not been explored. Collectively our data provide support for vaccine approaches based on multiple protective epitopes delivered either sequentially or in parallel.

While we have principally used whole parasite vaccination with PfSPZ Vaccine (in humans) or $\mathrm{Pb}$-PfCSP SPZ in mice for modelling antibody and B cell responses, the data herein may also explain some of the features of such responses to subunit vaccines for malaria. Notably in strong 351 agreement with our data it has previously been reported that the subunit RTS,S vaccine induces

352 anti-repeat responses that plateau after 2 immunizations, but responses to the Hepatitis B S

353 antigen core of the subunit continue to rise upon a third dose ${ }^{6,7}$. Our data also show that

354 boosting principally induces a short-lived PB response, which may temporarily allow the serum

355 titers of anti-(NANP) $)_{\mathrm{n}}$ antibodies to achieve protective levels; however, only a small fraction of

356 responding cells differentiate to become long-lived BMPCs which may explain the short-lived

357 protection by RTS,S even when additional late booster responses are given ${ }^{4,5}$. Understanding 358 how to induce a larger number of long-lived BMPCs will thus be a critical challenge for future 
vaccination approaches. Interestingly we found that delayed boosting resulted in secondary GCs

360 and higher antibody responses, which is consistent with findings that delayed administration of

361 the RTS,S vaccine results in better maturation of the B cell response and protection than a series

362 of boosts administered close together ${ }^{47}$.

364 Our data may have implications for vaccines targeting pathogens other than malaria. It has been

365 shown that responses to seasonal Influenza vaccination are inversely proportional to pre-existing

366 anti-Influenza titers suggesting a role for antibody feedback in limiting responses ${ }^{48}$. The

367 seasonal Influenza vaccine is a trivalent vaccine containing the HA molecule from three

368 circulating strains. When a novel trimeric cocktail is used for immunization that differs in some

369 components from the previous vaccine, responses are more robust to the divergent antigens,

370 consistent with a role for antibody feedback driving the diversification of the immune response

$371 \quad{ }^{49}$. For HIV, passive transfer studies in humans and vaccination studies in non-human primates

372 suggest that concentrations of broadly neutralising antibodies (bnAbs) will be required to confer

373 protection $3,50,51,52$. An additional problem is that bnAbs are highly mutated and hard to elicit by

374 vaccination, current vaccine approaches therefore focus on giving a series of antigenically

375 distinct Env trimers designed to stimulate germline precursors of bnAb producing plasma cells

376 and focus the somatic hypermutation process ${ }^{53}$. Antibody feedback may act as a double edge

377 sword, simultaneously limiting overall responses but stimulating the diversification of the

378 response at each step.

379

380 One striking finding is the mismatch between the amount of antibody required for protection and

381 the amount required for feedback. In naïve mice, feedback inhibition was observed at serum 
382 concentrations of antibody of $1-10 \mu \mathrm{g} / \mathrm{ml}$ anti-PfCSP, which is equivalent to $\sim 7-70 \times 10^{-9} \mathrm{M}$,

383 which is a little above the previously reported $\mathrm{K}_{\mathrm{d}}$ of $2 \mathrm{~A} 10$ for $\operatorname{PfCSP}\left(2.7 \times 10^{-9} \mathrm{M}\right){ }^{13}$. This

384 would imply that antibodies can efficiently mask the presentation of their cognate epitopes to B

385 cells at concentrations a little above their dissociation constant $\left(\mathrm{K}_{\mathrm{d}}\right)$. It is thus surprising that that

386 serum concentrations of $2 \mathrm{~A} 10$ of $\sim 200 \mu \mathrm{g} / \mathrm{ml}\left(>1 \times 10^{-6} \mathrm{M}\right)$ do not fully protect. This indicates

387 that simple binding of antibody is insufficient for high-level protection. Thus, protective

388 antibodies require some biological activity - either blocking the motility of sporozoite or

389 blocking CSP function - to exert protection ${ }^{20}$. One mechanism that may mitigate the low

390 amount of antibody that inhibits B cells responses may be the induction of memory T cell help.

391 Interestingly, in our experiments, antibody feedback appears less potent in an immune

392 background compared to in naive mice. This indicates that there is help for memory B cells in

393 immune mice that is not present in naïve mice, which is most likely attributable to T cells.

394 Nonetheless the specificity and induction of follicular helper T cells by sporozoite vaccines has

395 not been well-studied.

397 Our studies have been facilitated by the use of novel Ig-knockin mouse to dissect the B cell

398 response to PfCSP. This tool permits adoptive transfer experiments and the tracking of memory

399 B cells which would otherwise be challenging. The $\operatorname{Igh}^{\mathrm{g} 2 \mathrm{~A} 10}$ mouse is designed to carry B cells of

400 endogenous affinity as it carries a germline-reverted $\mathrm{IgH}_{\mathrm{H}} \mathrm{DJ}_{\mathrm{H}}$ rearrangement, which is free to

401 pair with any light chain. Notably our estimate of the affinity of our cells for PfCSP $\left(1.33 \times 10^{-7}\right.$

$402 \mathrm{M})$ is more than 4 orders of magnitude lower than the affinity of MD4 mice $\left(\sim 5 \times 10^{-12} \mathrm{M}\right)$ for

403 their cognate antigen HEL determined by others using a similar approach ${ }^{54}$. Our Igh ${ }^{\mathrm{g} 2 \mathrm{~A} 10}$-knockin

404 mouse also undergoes class switching and affinity maturation in a manner that appears to mirror 
405 these processes in human B cells. Due to the physiological nature of this mouse model we

406 anticipate it will be a useful tool in future studies of the B cell response to PfSPZ and different

407 vaccine modalities.

408

409 While antibody feedback is a well-established immunological phenomenon, its role in regulating

410 vaccine induced responses has not been clearly dissected. Antibody feedback will probably be a

411 critical challenge to the development of any vaccine where sustained high titers of neutralising

412 antibody are required for protection. Collectively our data explain some of the challenges facing

413 future vaccine development, but also offer some insights into how these challenges may be

414 overcome. 


\section{Materials and Methods}

417 Study subjects and clinical specimens.

VRC 314 clinical trial (https://clinicaltrials.gov/; NCT02015091) ${ }^{10,11}$ was an an open-label

420 evaluation of the safety, tolerability, immunogenicity and protective efficacy of Sanaria ${ }^{\circledR} \operatorname{PfSPZ}$

421 Vaccine. Subjects, recruited at the University of Maryland, Baltimore in the high dose cohort

422 received a total of three doses of 9x105 PfSPZ intravenously at week 0,8 and 16 . Blood was

423 drawn at the time of each immunization, as well as $7 \mathrm{~d}$ and $14 \mathrm{~d}$ after each immunization. Plasma

424 and PBMCs were isolated from all samples at these timepoints.

Isolation of plasmablasts.

PBMCs isolated from blood samples collected $7 \mathrm{~d}$ after immunization with PfSPZ Vaccine were used fresh or frozen and thawed prior to staining for viability with Aqua LIVE/DEAD dye

430 (Invitrogen) followed by surface staining and FACs sorting (full details of antibodies are given in

431 Supplementary Table 1). PBs were gated according to Supplementary Figure 1A and sorted as

432 single cells into 96-well PCR plates containing $20 \mu \mathrm{l} /$ well of reverse transcriptase reaction buffer

433 that included $5 \mu 1$ of $5 \times$ first-strand cDNA buffer, $0.5 \mu 1$ of RNAseOut (Invitrogen), $1.25 \mu 1$ of

434 dithiothreitol (DTT), $0.0625 \mu \mathrm{l}$ of igepal and $13.25 \mu \mathrm{l}$ of distilled $\mathrm{H}_{2} \mathrm{O}$ (Invitrogen) as previously 435 described $^{21}$.

Production of recombinant immunoglobulins. 
439 Immunoglobulin-encoding genes of PBs were amplified through RT and nested PCR without

440 cloning from RNA of single sorted cells as previously described ${ }^{19,21}$. The amplified rearranged

441 gene segments encoding variable regions were assembled into the corresponding linear full-

442 length immunoglobulin heavy- and light-chain gene expression cassettes through PCR as

443 previously described ${ }^{19,21}$. Heavy and light chain linear cassettes were co-transfected in $293 \mathrm{~T}$

444 cells using Effectene with enhancer (Qiagen) $)^{19,21}$. Transfected cultures were incubated at $37^{\circ} \mathrm{C}$

$4455 \% \mathrm{CO}_{2}$ for $3 \mathrm{~d}$. Supernatants were harvested, concentrated and purified using HiTrap Protein A

446 prepacked high-performance plates (GE Healthcare) for $20 \mathrm{~min}$ at room temperature on a shaker.

447 Following wash with PBS and $\mathrm{NaCl}$, eluates were neutralized with Trizma hydrochloride and

448 buffer exchanged with PBS before determining antibody concentration using Nanodrop.

449 Immunogenetic information was assigned to antibody sequences using Cloanalyst ${ }^{55}$ based on the

450 IMGT immunoglobulin gene segment libraries (http://www.imgt.org $)^{56}$. Sequences were

451 deemed to be clonally related using Cloanalyst ${ }^{55}$ based on their V and J gene usage and CDR3

452 similarity.

456 Recombinant monoclonal antibodies were screened for PfCSP reactivity using either ELISA or 457 electrochemiluminescence via the mesoscale discovery (MSD) platform. For ELISA MaxiSorp 458 ELISA plates (Thermo Scientific Nunc) were coated with $100 \mu \mathrm{l}$ of $\mathrm{rPfCSP}(1 \mu \mathrm{g} / \mathrm{ml})$ per well 459 for $1 \mathrm{~h}$ at room temperature according to the manufacturer's instructions (KPL). Coated plates 460 were blocked with $100 \mu \mathrm{l}$ of $1 \times$ blocking solution for $1 \mathrm{~h}$ at room temperature, which was 
461 followed by incubation with $100 \mu \mathrm{l}$ of PfCSP monoclonal antibodies, mock transfection filtrate

462 or control antibodies (VRC 01, a human anti-HIV-1 IgG1 as an isotype-matched negative

463 control $^{57} ; 2 \mathrm{~A} 10$, a mouse monoclonal antibody specific for the (NANP) $)_{\mathrm{n}}$-repeat region of

$\left.464 \operatorname{PfCSP}^{24,58}\right)$ at varying concentrations $(0.00006-5.0 \mu \mathrm{g} / \mathrm{ml})$. After $1 \mathrm{hr}$, plates were incubated

465 with $100 \mu \mathrm{l} /$ well of $1.0 \mu \mathrm{g} / \mathrm{ml}$ peroxidase-labeled goat anti-human IgG antibody (KPL). Plates

466 were washed six times with PBS-Tween between each step. After a final wash, samples were

467 incubated for about 15 min with the ABTS peroxidase (KPL) or Ultra TMB ELISA (Invitrogen)

468 substrate. The optical density was read at 405 or $450 \mathrm{~nm}$ after addition of stopping solution (100

$469 \mu \mathrm{l} /$ well).

471 Mesoscale Discovery (MSD) ELISA for PfCSP sera titers and screening of recombinant

472 monoclonal antibodies.

473

474 Streptavidin MSD 384 well plates (MSD) were first blocked with PBS + 5\%BSA for 30min,

475 washed five times, then coated with biotinylated antigen PfCSP-biotin, NANP Repeat-Biotin,

476 NTerm-Biotin, or CTerm-Biotin) at $1 \mu \mathrm{g} / \mathrm{mL}$ in PBS $+1 \%$ BSA. After $1 \mathrm{hr}$, plates were washed

477 and $10 \mu \mathrm{l}$ of serially diluted sera (starting at 1:10, then by 5 fold dilutions) in PBS $+0.05 \%$

478 Tween-20 + 1\%BSA, was added and incubated for $1 \mathrm{hr}$. Alternatively, for recombinant antibody

479 screening antibody concentrations were normalized to $1 \mu \mathrm{g} / \mathrm{ml}$ in PBS $+0.05 \%$ Tween-20 $+1 \%$

480 BSA prior to loading onto plates. After washing, plates were incubated for $1 \mathrm{hr}$ with sulfo-tag

481 goat anti-human $\mathrm{IgG}$ detection antibody (MSD) at $1 \mathrm{ug} / \mathrm{mL}$ diluted in PBS, $0.05 \%$ Tween,

482 1\%BSA. Plates were washed, and 1x Read T buffer (MSD) diluted in distilled water was added

483 before analyzing with MSD SECTOR Imager 2400. The log of mean fluorescence intensity 
484 (MFI) is reported. All incubations were done at room temperature and all wash steps were 485 performed 5 times.

Mice; generation of $\operatorname{Igh}^{g 2 A 10}$ knockin animals

489 C57BL/6NCrl were purchased from the Australian Phenomics Facility (Canberra, ACT, 490 Australia). MD4 mice (C57BL/6-Tg(IghelMD4)4Ccg; MGI:2384162) and Blimp1 GFP/+

491 (C57BL/6(Prdm 1 $1^{\text {tm1Nutt; }}$ MGI: 3510704) mice were a kind gift from Carola Vinuesa (The 492 Australian National University). FLPe deleter mice (B6.Cg-Tg(ACTFLPe)9205Dym/J; MGI:

$4933714491)^{59}$ were imported from Jackson laboratories (Bar Harbor, ME; stock number 005703).

$494 \operatorname{Igh}^{\mathrm{g} 2 \mathrm{~A} 10}$ were generated by Ozgene Pty Ltd (Bentley, WA, Australia) via embryonic cell

495 transformation. Briefly the predicted germline precursor gene of the heavy chain of the 2A10

496 antibody was synthesised and inserted into a plasmid carrying flanking regions corresponding to 497 positions chr12:113430554 to chr12:113435542 (for the 5'homology arm) and chr12:113425551 498 to chr12:113428513 (for the 3' homology arm) for targeting into the IgM locus. Upstream of the 499 g2A10 gene was an Igh promoter and a neomycin cassette flanked by Frt sites for subsequent 500 excision. Subsequently mice were crossed to FLPe deleter mice which constitutively express 501 FLPe under the control of the actin promoter to generate mice $\operatorname{Igh}^{\mathrm{g} 2 \mathrm{~A} 10}$ mice lacking the 502 Neomycin cassette. Mice were bred and maintained under specific pathogen free conditions in 503 individually ventilated cages at the Australian National University. All animal procedures were 504 approved by the Animal Experimentation Ethics Committee of the Australian National 505 University (Protocol numbers: A2013/12 and A2016/17). All mice were 5-8 weeks old at the 
commencement of experiments. Within each experiment, mice were both age matched. Female

507 mice were used throughout the experiments.

Immunizations and Antibody Transfer

511 Mice were immunized IV with $5 \times 10^{4} \mathrm{~Pb}$-PfCSP SPZ crossed to an mCherry background to

512 facilitate the identification of infected mosquitoes ${ }^{27,60}$. Sporozoites were dissected by hand from

513 the salivary glands of Anopheles stephensi mosquitoes and were irradiated (200kRad) using a

514 MultiRad 225 (Flaxitron) irradiator prior to injection. For PfCSP immunization, 30 $\mu$ g rPfCSP ${ }^{13}$

515 in PBS was absorbed with Imject Alum (ThermoFisher) in a 2:1 ratio of antigen: adjuvant

516 according to the manufacturer's instructions and injected IP in a final volume of $150 \mu 1$.

518 Antibodies for passive transfer were injected IV at the stated doses. 5D5 ${ }^{41}$ (mouse IgG1) was a

519 kind gift of Gabriel Gutierrez (Leidos). $\mathrm{CIS}^{20} 3^{20}, \mathrm{VRC} 1^{57}$ and $\mathrm{mAb} 15^{20}$ (all human IgG1), were

520 expressed in-house at the Vaccine Research Center from Expi293T cells. 2A10²4, 58 (mouse

521 IgG2a) was prepared from hybridoma cell supernatants (Genscript). 2A10 LALA-PG antibodies

522 carrying L234A, L235A and P329G substitutions in the IgG2A heavy chain $^{38}$ were expressed

523 from Expi293F cells (Genscript). LTF-2 (mouse IgG2b) was purchased from BioXCell.

525 Kinetic binding assay using biolayer interferometry.

526 Antibody binding kinetics were performed using biolayer interferometry on an Octet Red384

527 instrument (fortéBio) using streptavidin-capture biosensors (fortéBio) as previously described ${ }^{20}$. 
528 PfCSP monoclonal antibody solutions were plated in solid black tilt-well 96-well plates (Geiger

529 Bio-One). Loading of biotinylated rPfCSP was performed for $300 \mathrm{~s}$, followed by dipping the

530 biosensors into buffer (PBS $+1 \% \mathrm{BSA}$ ) for $60 \mathrm{~s}$ to assess baseline assay drift. Association with

531 whole IgG (serially diluted from 33 to $0.5208 \mu \mathrm{M}$ ) was done for $300 \mathrm{~s}$, followed by a

532 dissociation step in buffer for $600 \mathrm{~s}$. Background subtraction of nonspecific binding was

533 performed through measurement of association in buffer alone. Data analysis and curve fitting

534 were performed using Octet software, version 7.0. Experimental data were fitted with the binding

535 equations describing a 1:1 heterologous ligand interaction. Global analyses of the complete data

536 sets, assuming binding was reversible (full dissociation), were carried out using nonlinear least-

537 squares fitting allowing a single set of binding parameters to be obtained simultaneously for all

538 concentrations of a given monoclonal antibody dilution series.

Flow Cytometry and sorting

542 Lymphocytes were isolated from the spleen and bone marrow of mice and were prepared into

543 single cell suspensions for flow cytometric analysis and sorting. Bone marrow cells were flushed

544 from femurs and tibias with FACs buffer in $27 \mathrm{~g}$ syringes, whilst splenocytes were isolated by

545 mashing spleens over $70 \mu \mathrm{m}$ micron mesh filters. Red blood cells were lysed from cell

546 suspensions with ACK lysis buffer (Sigma) and cells were washed twice with FACs wash prior

547 to antibody staining. Cells were quantified during flow cytometry by the addition of CountBright

548 Absolute Counting beads (Invitrogen) to sample suspensions. Details of antibodies are given in

549 Supplementary Table 1, and details of generic gating strategies for mouse experiments are given

550 in Supplementary Figure 2. (NANP) 9 tetramers were prepared in house by mixing biotinylated 
551 (NANP) 9 peptide with streptavidin conjugated PE or APC (Invitrogen) in the a 4:1 molar ratio.

552 Flow-cytometric data was collected on a BD Fortessa or X20 flow cytometer (Becton Dickinson)

553 and analyzed using FlowJo software (FlowJo). A BD FACs Aria I or II (Becton Dickinson)

554 machine was used for FACS sorting of cells.

Cell purification and adoptive transfers

For primary immunizations the number of naïve tetramer ${ }^{+}$cells were quantified from Igh $^{\text {g2A10 }}$ splenocytes via flow cytometry. The concentration of donor splenocytes were then adjusted to deliver 1-2 x $10^{4}$ tetramer $^{+}$Igh $^{\mathrm{g} 2 \mathrm{~A} 10} \mathrm{CD} 19+\mathrm{B}$ cells to each recipient mouse in $100 \mu \mathrm{l}$ via IV

561 injection. Mice were immunized 1-2 days after adoptive transfer of naïve splenocytes.

563 Memory cells for adoptive transfer experiments were enriched from mice that had received naïve

$564 \operatorname{Igh}^{\mathrm{g} 2 \mathrm{~A} 10} \mathrm{~B}$ cells and been immunized with either Pb-PfCSP SPZ or PfCSP. 8-10 weeks after

565 immunization, memory cell donor mice were culled and single cell preparations of lymphocytes

566 from the spleen were made. Splenocytes were treated with FC-block (anti-CD16/32, Biolegend)

567 and then incubated with lineage specific biotin conjugates (anti-GL7, anti-CD138, anti-CD4, and

568 anti-CD8; see supplementary table 1 for details of clones and suppliers) to remove T cells, PBs

569 and germinal centre B cells. Afterwards, splenocytes were incubated with anti-biotin microbeads

570 (Miltenyi), and then put through a magnetic LS column (Miltenyi), according to the

571 manufacturer's directions. A cell suspension enriched in Igh $^{\mathrm{g} 2 \mathrm{~A} 10}$ memory B cells was collected

572 in the runoff after passing through the column (Supplementary Figure 5). A fraction of the

573 sample was stained for flow cytometric analysis to quantify the number of CD45. $1^{+} \mathrm{Tet}^{+}$memory 
574 cells, and to confirm that depletion of other B cell subsets and helper T cells had worked. The

575 concentration of cells was adjusted such that $5 \times 10^{2} \mathrm{Igh}^{\mathrm{g} 2 \mathrm{~A} 10}$ memory cells were then transferred

576 to recipient mice IV in a volume of $100 \mu l$.

577

ELISA for the detection of anti-PfCSP antibodies in mice

Concentrations of PfCSP specific antibodies in the sera of mice after immunization were

581 measured using solid phase ELISA. Briefly, Nunc Maxisorp Plates (Nunc-Nucleon) were coated

582 overnight with $1 \mathrm{ug} / \mathrm{ml}$ streptavidin followed by binding of biotinylated (NANP) 9 peptide for 1

583 hour. After blocking with 1\% BSA, serial dilutions of the antibodies were incubated on the plates

584 for 1 hour and after washing, incubated with HRP conjugated anti-mouse IgG or anti-mouse IgM

585 antibodies (KPL). Plates were developed for 15-20 minutes with ABTS 2-Component Peroxidase

586 Substrate Kit (KPL), and read at 405nm using a Tecan Infinite 200Pro plate reader. IgM

587 responses were analysed as the area under the absorbance curve (AUC). IgG concentrations were

588 calculated from a standard curve that was generated from serial dilutions of 2A10 (starting at

$5891 \mu \mathrm{g} / \mathrm{ml})$.

591 To assess circulating levels of passively transferred PfCSP-specific monoclonal antibodies, mice

592 were bled via the tail plexus immediately before challenge with infectious mosquito bites.

593 ELISA was performed on mouse serum as previously described using plates coated as above. A

594 standard curve for each monoclonal antibody was generated using eight 3-fold dilutions of

595 monoclonal antibody starting at $1 \mu \mathrm{g} / \mathrm{ml}$. Serum samples were applied at a series of dilutions

596 from 1:500-1:4500 in blocking buffer. The concentrations were commonly calculated off the 
597 values from the $\sim 1: 1500$ dilution. However, if the sample was uncommonly low/high then other

598 dilutions were used for the calculation (providing they sat within the exponential range of the

599 monoclonal standard curve).

600

ELISpot for the detection of PfCSP specific plasmablasts

602

603 Sterile MultiScreen ${ }^{\mathrm{TM}}$-HA plates (Millipore) were coated with PfCSP at $2.5 \mu \mathrm{g} / \mathrm{ml}$ in PBS, and

604 left overnight at 4C. After washing with sterile PBS, plates were blocked with complete RPMI

6051640 (10\% FCS, 2 mM L-glutamine, 1 mM Na-Pyruvate, 100 U/ml Penicillin/Streptomicin,

$6065 \mathrm{mM}$ HEPEs, $20 \mu \mathrm{g} / \mathrm{ml}$ Gentamicin and $50 \mu \mathrm{M} \beta$-mercaptoethanol) for 3 hours at $37 \mathrm{C}$. Sorted

607 bone marrow derived plasma cells were added to selected wells, and incubated overnight at 37C.

608 The following day plates were washed with wash buffer, and incubated for 3 hours with HRP

609 conjugated anti-mouse IgG (KPL). After washing with wash buffer, plates were developed using

610 stable DAB (Invitrogen) for 20 minutes. The number of spots was counted manually in each well

611 by two individuals blinded to the experimental groups.

In vivo protection in $C 57 B L / 6$ mice with chimeric Pb-PfCSP SPZ.

615 For the mosquito bite challenge, female $A$. stephensi mosquitoes were allowed to feed on 8-

616 week-old C57BL/6 mice infected with blood-stage Pb-PfCSP-mCherry. 21 days after feeding

617 mosquitoes were chilled on ice and sorted for infection. The abdomens and thoraxes of infected

618 mosquitoes glow red under green fluorescent light due to the presence of mCherry ${ }^{+}$midgut and

619 salivary gland sporozoites facilitating sorting. Mice were challenged with $\sim 5$ infected 
620 mosquitoes per mouse. C57BL/6 mice were injected IV with monoclonal antibodies as stated in

621 the relevant figure legends. Ten minutes later, mice were anesthetized with Ketamine-Xylazine

$622(100 \mathrm{mg} / \mathrm{kg}$ and $10 \mathrm{mg} / \mathrm{kg}$ respectively), and the infected mosquitoes were allowed to feed on

623 mice for $\sim 30 \mathrm{~min}$, after which mosquito abdomens were visually inspected for blood, indicating

624 the mosquito has bitten. Mouse parasitemia was assessed daily through flow cytometry from day

6254 through day 14 post-infection. A mouse was considered patent once parasitemia reached

$626>0.1 \%$.

627

Single cell RNA-seq to sequence recombined Ig V(D)J chains from mice

630 Single Cell RNA sequencing was performed using a SMARTseq 2 protocol ${ }^{33}$ with the following

631 modifications. Cells were sorted into plates with wells containing 1 ul of the cell lysis buffer, 0.5

$632 \mu \mathrm{ldNTP} \operatorname{mix}(10 \mathrm{mM})$ and $0.5 \mu \mathrm{l}$ of the oligo-dT primer at $5 \mu \mathrm{M}$. We then reduced the amount

633 reagent used in the following reverse-transcription and PCR amplification step by half. The

634 concentration of the IS PCR primer was also further reduced to $50 \mathrm{nM}$. Due to the low

635 transcriptional activity of memory B cells, we increased the number of PCR cycles to 28.

636 Sequencing libraries were then prepared using the Nextera XT Library Preparation Kit with the

637 protocol modified by reducing the original volumes of all reagents in the kit by $1 / 5^{\text {th }}$. Sequencing

638 was performed on the Illumina NextSeq sequencing platform.

639 To determine the antigen-specific BCR repertoire, we made use of VDJpuzzle ${ }^{61}$ to reconstruct

640 full-length heavy and light chains from each cell. From this we were able to determine V region

641 usage and mutation frequency.

642 
Experimental design and statistical analysis

644

645 Details of specific statistical tests and experimental design are given in the relevant figure

646 legends. Mouse experiments had 3-5 mice per group and were performed either in duplicate or

647 triplicate. All data points are plotted from all replicate experiments, though for one human

648 subject (figure 1B/7B)) data were excluded from subsequent statistical analysis as these

649 individuals did not respond to immunization; excluded data points are marked by \#. In most

650 instances analysis was performed in R (The R Foundation for Statistical Computing) on the

651 pooled data from all replicate experiments. Where data was pooled from multiple experiments,

652 each experiment was included as a blocking factor in the analysis. Where data are plotted on a

653 log-scale data were log-transformed prior to analysis. For certain experiments and the analysis of

654 some human data blocking factors did not need to be accounted for and analysis was performed

655 in GraphPad Prism 7. Abbreviations for $\mathrm{p}$ values are as follows: $\mathrm{p}<0.05=*, \mathrm{p}<0.01=* *, \mathrm{p}<$

$6560.001=* * *, \mathrm{p}<0.0001=* * * *$; with only significant $\mathrm{p}$ values shown. With the exception of

657 ELISpot counting, no blinding or randomization was performed, however all other readouts

658 (ELISA, flow cytometry and sequencing) are objective readouts that are not subject to

659 experimental bias. 


\section{Author Contributions}

661

662 Conceptualization: H.A.M., A.H.I., R.A.S., I.A.C.; Investigation: H.A.M., A.H.I., H.J.S., B.J.F.,

663 Y.C., D.C., K.L., S.C., N.K., B.K.L.S., M.B., I.A.C.; Formal analysis: H.A.M., A.H.I., H.J.S.,

664 K.W., M.B., I.A.C.; Resources: S.L.H.; Writing-original draft preparation: H.A.M. and I.A.C.;

665 Writing - review and editing: A.H.I., M.B. and R.A.S.. Project administration R.A.S. and I.A.C.;

666 Funding acquisition: S.L.H., R.A.S. and I.A.C.

667

668

669 Competing Interests

670

671 S.C., N.K., B.K.L.S., and S.L.H. are salaried employees of Sanaria Inc., the developer and owner

672 of PfSPZ Vaccine and the investigational new drug (IND) application sponsor of the clinical

673 trials. S.L.H. and B.K.L.S. have a financial interest in Sanaria Inc. All other authors declare no

674 conflict of interest.

675

676

677 Acknowledgements

678

679 This work was supported by start-up funds from the Australian National University to I.A.C. and

680 NHMRC project grant support to I.A.C. (GNT1158404). Production and characterization of

681 PfSPZ Vaccine were supported in part by National Institute of Allergy and Infectious Diseases

682 Small Business Innovation Research Grants 5R44AI055229-11 (to S.L.H.), 5R44AI058499-08 
683 (to S.L.H.), and 5R44AI058375-08 (to S.L.H.). We would like to thank the University of

684 Maryland study volunteers from malaria clinical trial VRC314. We would like to that Harpreet

685 Vohra and Michael Devoy of the Imaging and Cytometry Facility at the Australian National

686 University for assistance with flow cytometry and sorting. We thank Morgan Gladden, Anthony

687 Monroe, Ruijun Zhang, Minyue Wang and Joshua Beem of the Duke Human Vaccine Institute

688 for assistance with gene amplification, antibody production and immunogenetics analysis.

689 Special thanks to Joe R. Francica for technical support and guidance with antibody affinity

690 measurements. We would like to acknowledge the support of the flow cytometry core at the

691 Vaccine Research Center particularly David Ambrozak for assistance with PB sorting. 


\section{Figure Legends}

693

Figure 1: Limited memory B cell responses to PfCSP following repeated vaccination.

695 A. Schematic of the vaccination protocol for the VRC314 clinical trial of PfSPZ with the timing

696 of sera and PBMC collection. B. Antibody responses to whole PfCSP measured by

697 electrochemiluminescence at the stated timepoints after each immunization, antibody responses

698 in individuals selected for downstream PB analysis are highlighted in colour; analysis was

699 performed by repeated measured one-way ANOVA with Tukey’s multiple comparisons test. C.

700 Proportion of PfCSP specific PBs isolated from 3 donors after each immunization segregated by

701 the use of the IGHV3.33 allele; each individual wedge indicates a unique clone. Analysis was

702 performed by chi-squared test with subject as a blocking factor. D. Mutational frequency in the

703 heavy chain gene of non-PfCSP specific PBs ( $\alpha$-other; grey circles) and PfCSP specific PBs ( $\alpha$ -

704 PfCSP; red triangles) after each immunization, bars show mean \pm s.d.; analysis by two-way

705 ANOVA with subject as a blocking factor. E. Manhattan plots showing the location and

706 frequency of mutations in the IGHV3.33 genes of non-PfCSP specific PB clones and PfCSP-

707 specific PB clones pooled from the three subjects sequenced. F. Frequency of different amino

708 acid changes at positions 32 and 55 in PfCSP and non-PfCSP specific PB clones.

710 Figure 2: Development of Igh ${ }^{\mathrm{g} 2 \mathrm{A10}}$ mice to track B cell responses to PfCSP.

711 A. Representative FACs plots (gated on B cells) showing the percentage of B cells that bind to

712 (NANP) ${ }_{9}$-tetramers in $\mathrm{C} 57 \mathrm{BL} / 6$ and $\operatorname{Igh}^{\mathrm{g} 2 \mathrm{~A} 10}$ mice, and the $\operatorname{IgM}$ and $\operatorname{IgD}$ expression on these

713 cells. B. Titration of the concentration of PfCSP required to block tetramer staining of Igh ${ }^{\mathrm{g} 2 \mathrm{~A} 10}$

714 cells; $\operatorname{Igh}^{\mathrm{g} 2 \mathrm{~A} 10}$ cells were incubated with the indicated concentrations of PfCSP, prior to 
715 (NANP)9-Tetramer staining and flow cytometry, analysis by non-linear regression. C. Schematic

716 of immunization experiment in which mice received Igh $^{\mathrm{g} 2 \mathrm{~A} 10}$ cells and were immunized with $\mathrm{Pb}-$

717 PfCSP SPZ (SPZ) or salivary gland extract (SGE). D. Concentration of anti-(NANP) $)_{\mathrm{n}}$ antibodies

718 in mice immunized as in $\mathrm{C}$, left axis represents $\mathrm{IgG}$ (expressed as $\mu \mathrm{g} / \mathrm{ml}$ ) and right axis

719 represents IgM expressed as area under the curve (AUC), means \pm s.d. shown. E. Representative

720 flow cytometry plots showing the phenotypes of $\operatorname{Igh}^{\mathrm{g} 2 \mathrm{~A} 10} \mathrm{~B}$ cells in mice immunized with $\mathrm{Pb}-$

721 PfCSP SPZ as outlined in C. F. Summary data showing the proportions of $\operatorname{Igh}^{\mathrm{g} 2 \mathrm{~A} 10}$ cells that

722 expressed $\operatorname{IgD}, \operatorname{IgM}$ or neither (SwIg) at the indicated timepoints; means \pm s.d. shown. G.

723 Summary data showing the proportions of $\operatorname{Igh}^{\mathrm{g} 2 \mathrm{~A} 10}$ cells that are PC, GC B cells or memory B

724 cells at the indicated timepoints; means \pm s.d. shown. H. Gating strategy for the identification

725 and sorting of $\operatorname{Igh}^{\mathrm{g} 2 \mathrm{~A} 10}$ BMPCs and representative PfCSP coated ELISpot wells probed with anti-

726 IgG-HRP from mice immunized as in C. I. Summary data based on H. showing the number of

727 CSP-specific Igh ${ }^{\mathrm{g} 2 \mathrm{~A} 10}$ antibody secreting cells per leg from 3 mice as identified by ELISpot, the $\mathrm{n}$

728 given above is the total number of cells in each gate that were sorted into each well.

Figure 3: Limited memory B cell recall responses to PfCSP following repeated vaccination

731 in mice.

732 A. Immunization schedule for the experiment: mice received $2 \times 10^{4}$ congenically marked

733 Igh $^{\mathrm{g} 2 \mathrm{~A} 10}$ Blimp $^{\mathrm{gfp} /+}$ cells and were immunized 1,2 or 3 times with $5 \times 10^{4} \mathrm{~Pb}$-PfCSP SPZ at 4

734 week intervals. 5 days after each boost and 33 days after the final boost blood, splenocytes and

735 bone marrow was collected from the mice for analysis by ELISA and flow cytometry. B.

736 Concentrations of anti-(NANP) $)_{n} \operatorname{IgG}$ in the sera at the indicated timepoints. C. Representative

737 flow cytometry plots for the identification of $\operatorname{Igh}^{\mathrm{g} 2 \mathrm{~A} 10} \mathrm{PBs}$ and plasma cells in the spleen. D. 
738 Representative flow cytometry plots for the identification of Igh $^{\mathrm{g} 2 \mathrm{~A} 10}$ GC B cells and memory

739 cells in the spleen. Summary data for the analysis of spleen PBs (E), spleen memory B cells (F),

740 spleen GC B cells $(\mathrm{G})$ and BMPCs $(\mathrm{H})$ at the indicated timepoints; data are pooled from 3

741 replicate experiments, analysis was by 2-way ANOVA including the experiment as a blocking

742 factor, bars represent means \pm s.d..

\section{Figure 4: Memory B cells induced by sporozoite immunization are able to mount recall}

responses. A. Schematic of the experiment showing the protocol for the generation and transfer of Igh ${ }^{\mathrm{g} 2 \mathrm{~A} 10}$ memory B cells to naïve recipient mice and subsequent boosting with Pb-PfCSP SPZ. after boosting with $5 \times 10^{4} \mathrm{~Pb}$-PfCSP SPZ compared to naïve mice that did not receive memory cells, but were immunized concurrently with $5 \times 10^{4}$ Pb-PfCSP SPZ; data from a single 
761 Representative flow cytometry plots of Igh $^{\mathrm{g} 2 \mathrm{~A} 10}$ memory cells recovered by magnetic bead

762 purification from recipient mice immunized as in $\mathrm{A}$. gated on $\mathrm{CD} 19^{+}$or $\mathrm{CD} 138^{+} \mathrm{B}$ cells and $\mathrm{PBs}$.

763 C. Quantification of the numbers of recovered cells in each group; data pooled from 2

764 experiments, bars show mean \pm s.d., with analysis by one-way ANOVA using Tukey’s multiple

765 comparisons test, with experiment as a blocking factor, only significant comparisons with the

766 positive control group ( $\mathrm{Pb}$-PfCSP boosted naïve $\mathrm{C} 57 \mathrm{BL} / 6$ recipients) are shown. D. Correlation

767 of the sera titers of anti-(NANP) $)_{\mathrm{n}}$ antibodies prior to boosting in the different groups of mice in

768 A. with the subsequent size of the expansion of the CD45.1 $1^{+}$Tetramer $^{+}$B cell population after

769 boosting; data pooled from 2 experiments, analysis by linear regression. E. Correlation of the

770 response (change in anti-PfCSP antibody level) to V2 and V3 boosts with the titer of antibodies

771 prior to the corresponding boost among the PfSPZ vaccinated individuals described in Figure

772 1A; analysis by linear regression.

773

Figure 6: Sub-protective levels of antibody potently inhibit memory B cell responses. A.

775 Survival plots showing the proportion of uninfected mice after IV transfer of the specified

776 amounts of 2 A10 antibody and feeding by 7 infected mosquitoes; data pooled from 2

777 experiments, analysis by Log-rank (Mantel-Cox) test showing pairwise comparisons with the no

778 antibody group. B. Concentration of 2A10 antibody in the blood 2 hours post-transfer via

779 ELISA; hollow circles indicate protected mice, means \pm s.d. shown. C. Expansion of Igh ${ }^{\mathrm{g} 2 \mathrm{~A} 10}$

780 memory B cells (generated using Pb-PfCSP SPZ immunization and boosted as in figure 4A) in

781 recipient mice that received the specified doses of $2 \mathrm{~A} 10$; data pooled from 2 independent

782 experiments and analysed by one-way ANOVA using Tukey's multiple comparisons test with

783 experiment as a blocking factor. D. Proportions of $\operatorname{Igh}^{\mathrm{g} 2 \mathrm{~A} 10}$ memory cells from $(\mathrm{C})$ that had 
784 differentiated into PBs, GC B cells, or retained a memory phenotype. E. Survival plots showing

785 the proportion of uninfected mice after IV transfer of the specified amounts of CIS43 antibody

786 and feeding by 7 infected mosquitoes; data pooled from 2 experiments, analysis by Log-rank

787 (Mantel-Cox) test showing pairwise comparisons with the no antibody group. F. Concentration

788 of CIS43 antibody in the blood 2 hours post-transfer via ELISA; hollow circles indicate

789 protected mice, means \pm s.d. shown. G. Expansion of memory B cells (generated using Pb-

790 PfCSP immunization and boosted as in figure 4A) in recipient mice that received $33 \mu \mathrm{g}$ of the

791 specified anti-CSP (2A10 and CIS43) antibodies, and antibodies of irrelevant specificity (LTF2

792 and VRC01); data pooled from 2 independent experiments and analysed by one-way ANOVA

793 using Tukey's multiple comparisons test with experiment as a blocking factor, means \pm s.d.

794 shown. H. Numbers of recovered PB, GC and memory CD45.1+ Tetramer+ cells from the no

795 antibody/no immunization, 2A10 and CIS43 groups in G; means \pm s.d. shown; analysis by two-

796 way ANOVA with experiment as a blocking factor.

797

Figure 7: Antibody feedback occurs via epitope masking and allows the diversification of

799 the antibody response. A. Schematic of the CSP molecule showing the binding sites and

800 dissociation constants of the different antibodies used in this study. B. Expansion of memory B

801 cells in the presence of antibodies (30 $\mathrm{g} / \mathrm{mouse})$ targeting non-repeat regions of CSP (5D5 and

$802 \mathrm{mAb} 15)$, anti-repeat Fc-dead 2A10 (2A10-LALA-PG) and 2A10 transferred 4 hours post

803 sporozoite delivery; memory cells were generated via Pb-PfCSP SPZ immunization and

804 transferred as in figure 4A and expansion measured 5 days post boosting with $5 \times 10^{4} \mathrm{~Pb}-\mathrm{PfCSP}$

805 SPZ. C. Expansion of memory B cells in the presence of anti-PfCSP antibodies at different

806 concentrations; memory cells were generated via Pb-PfCSP SPZ immunization and transferred 
807 as in figure $4 \mathrm{~A}$ and expansion measured 5 days post boosting with $5 \times 10^{4} \mathrm{~Pb}-\mathrm{PfCSP} \mathrm{SPZ}$. D.

808 Antibody responses specific for the (NANP) $)_{n}$-repeat and C-terminal domain of CSP in PfSPZ

809 vaccinated subjects (described in Figure 1A) analysis was performed by repeated measures one-

810 way ANOVA with Tukey's multiple comparisons test; \# indicates one individual who did not

811 respond and was excluded from subsequent analysis. F. Correlation of the response (change in

812 anti-(NANP)n antibody level) to V2 and V3 boosts with the titer of antibodies prior to the

813 corresponding boost; analysis by linear regression. G. Correlation of the response (change in

814 anti-terminal antibody level) to V2 and V3 boosts with the titer of antibodies prior to the

815 corresponding boost; analysis by linear regression. 


\section{References}

818 1. Plotkin, S.A. Vaccines: correlates of vaccine-induced immunity. Clinical infectious

819 diseases : an official publication of the Infectious Diseases Society of America 47, 401-

$820 \quad 409(2008)$.

821

822

2. Amanna, I.J., Carlson, N.E. \& Slifka, M.K. Duration of humoral immunity to common

823

824

825

826

827

828

829

830

831

832

833

834 viral and vaccine antigens. The New England journal of medicine 357, 1903-1915 (2007).

3. Pauthner, M.G. et al. Vaccine-Induced Protection from Homologous Tier 2 SHIV Challenge in Nonhuman Primates Depends on Serum-Neutralizing Antibody Titers. Immunity 50, 241-252 e246 (2019).

4. White, M.T. et al. The relationship between RTS,S vaccine-induced antibodies, CD4(+) $\mathrm{T}$ cell responses and protection against Plasmodium falciparum infection. PloS one $\mathbf{8}$, e61395 (2013).

6. Kester, K.E. et al. Phase 2a trial of 0,1 , and 3 month and 0 , 7, and 28 day immunization schedules of malaria vaccine RTS,S/AS02 in malaria-naive adults at the Walter Reed Army Institute of Research. Vaccine 26, 2191-2202 (2008).

7. Kester, K.E. et al. Efficacy of recombinant circumsporozoite protein vaccine regimens against experimental Plasmodium falciparum malaria. The Journal of infectious diseases 183, 640-647 (2001).

845

8. Seder, R.A. et al. Protection against malaria by intravenous immunization with a nonreplicating sporozoite vaccine. Science 341, 1359-1365 (2013).

9. Epstein, J.E. et al. Live attenuated malaria vaccine designed to protect through hepatic

10. Ishizuka, A.S. et al. Protection against malaria at 1 year and immune correlates following PfSPZ vaccination. Nature medicine 22, 614-623 (2016). (2017). 
12. Dups, J.N., Pepper, M. \& Cockburn, I.A. Antibody and B cell responses to Plasmodium sporozoites. Frontiers in microbiology 5, 625 (2014).

13. Fisher, C.R. et al. T-dependent B cell responses to Plasmodium induce antibodies that form a high-avidity multivalent complex with the circumsporozoite protein. PLoS Pathog 13, e1006469 (2017).

14. Keitany, G.J. et al. Immunization of mice with live-attenuated late liver stage-arresting Plasmodium yoelii parasites generates protective antibody responses to preerythrocytic stages of malaria. Infection and immunity 82, 5143-5153 (2014).

15. Murugan, R. et al. Clonal selection drives protective memory B cell responses in controlled human malaria infection. Science immunology 3 (2018).

16. Mordmuller, B. et al. Sterile protection against human malaria by chemoattenuated PfSPZ vaccine. Nature 542, 445-449 (2017).

17. Olotu, A. et al. Seven-Year Efficacy of RTS,S/AS01 Malaria Vaccine among Young African Children. The New England journal of medicine 374, 2519-2529 (2016).

18. RTS,S Clinical Trials Partnership et al. A phase 3 trial of RTS,S/AS01 malaria vaccine in African infants. The New England journal of medicine 367, 2284-2295 (2012).

19. Bonsignori, M. et al. Analysis of a clonal lineage of HIV-1 envelope V2/V3 conformational epitope-specific broadly neutralizing antibodies and their inferred unmutated common ancestors. J Virol 85, 9998-10009 (2011).

20. Kisalu, N.K. et al. A human monoclonal antibody prevents malaria infection by targeting a new site of vulnerability on the parasite. Nature medicine (2018).

21. Liao, H.X. et al. High-throughput isolation of immunoglobulin genes from single human B cells and expression as monoclonal antibodies. J Virol Methods 158, 171-179 (2009).

22. Oyen, D. et al. Cryo-EM structure of P. falciparum circumsporozoite protein with a vaccine-elicited antibody is stabilized by somatically mutated inter-Fab contacts. Sci $A d v$ 4, eaau8529 (2018).

23. Anker, R., Zavala, F. \& Pollok, B.A. VH and VL region structure of antibodies that recognize the (NANP)3 dodecapeptide sequence in the circumsporozoite protein of Plasmodium falciparum. Eur J Immunol 20, 2757-2761 (1990).

24. Nardin, E.H. et al. Circumsporozoite proteins of human malaria parasites Plasmodium falciparum and Plasmodium vivax. The Journal of Experimental Medicine 156, 20-30 (1982). 
25. Yang, Y. et al. Distinct mechanisms define murine B cell lineage immunoglobulin heavy chain (IgH) repertoires. Elife 4, e09083 (2015).

26. Kaplinsky, J. et al. Antibody repertoire deep sequencing reveals antigen-independent selection in maturing B cells. Proceedings of the National Academy of Sciences of the United States of America 111, E2622-2629 (2014).

27. Espinosa, D.A. et al. Robust antibody and CD8(+) T-cell responses induced by P. falciparum CSP adsorbed to cationic liposomal adjuvant CAF09 confer sterilizing immunity against experimental rodent malaria infection. NPJ vaccines 2 (2017).

28. Shih, T.A., Meffre, E., Roederer, M. \& Nussenzweig, M.C. Role of BCR affinity in T cell dependent antibody responses in vivo. Nat Immunol 3, 570-575 (2002).

29. Suan, D. et al. T follicular helper cells have distinct modes of migration and molecular signatures in naive and memory immune responses. Immunity 42, 704-718 (2015).

30. Manz, R.A., Thiel, A. \& Radbruch, A. Lifetime of plasma cells in the bone marrow. Nature 388, 133-134 (1997).

31. Slifka, M.K., Matloubian, M. \& Ahmed, R. Bone marrow is a major site of long-term antibody production after acute viral infection. J Virol 69, 1895-1902 (1995).

32. Kallies, A. et al. Plasma cell ontogeny defined by quantitative changes in blimp-1 expression. J Exp Med 200, 967-977 (2004).

33. Picelli, S. et al. Full-length RNA-seq from single cells using Smart-seq2. Nat Protoc 9, 171-181 (2014).

34. Zuccarino-Catania, G.V. et al. CD80 and PD-L2 define functionally distinct memory B cell subsets that are independent of antibody isotype. Nat Immunol 15, 631-637 (2014).

35. Goodnow, C.C., Crosbie, J., Jorgensen, H., Brink, R.A. \& Basten, A. Induction of selftolerance in mature peripheral B lymphocytes. Nature 342, 385-391 (1989).

36. Ravetch, J.V. Fc receptors: rubor redux. Cell 78, 553-560 (1994).

37. Heyman, B. Antibody feedback suppression: towards a unifying concept? Immunol Lett 68, 41-45 (1999).

38. Lo, M. et al. Effector-attenuating Substitutions That Maintain Antibody Stability and Reduce Toxicity in Mice. J Biol Chem 292, 3900-3908 (2017).

39. Radtke, A.J. et al. Lymph-node resident CD8alpha+ dendritic cells capture antigens from migratory malaria sporozoites and induce CD8+ T cell responses. PLoS Pathog 11, e1004637 (2015). 
953

954

955

956

957

958

959

960

961

962

963

964

965

966

967

968

969

970

971

972

973

974

975

976

977

978

979

980

981

982

983

984

985

986

987

988

989

990

991

992

993

994

40. Yamauchi, L.M., Coppi, A., Snounou, G. \& Sinnis, P. Plasmodium sporozoites trickle out of the injection site. Cell Microbiol 9, 1215-1222 (2007).

41. Espinosa, D.A. et al. Proteolytic Cleavage of the Plasmodium falciparum Circumsporozoite Protein Is a Target of Protective Antibodies. The Journal of infectious diseases 212, 1111-1119 (2015).

42. Dobano, C. et al. Concentration and avidity of antibodies to different circumsporozoite epitopes correlate with RTS,S/AS01E malaria vaccine efficacy. Nat Commun 10, 2174 (2019).

43. Ubillos, I. et al. Baseline exposure, antibody subclass, and hepatitis B response differentially affect malaria protective immunity following RTS,S/AS01E vaccination in African children. BMC Med 16, 197 (2018).

44. Grantham, W.G. \& Fitch, F.W. The role of antibody feedback inhibition in the regulation of the secondary antibody response after high and low dose priming. J Immunol 114, 394398 (1975).

45. Karlsson, M.C., Wernersson, S., Diaz de Stahl, T., Gustavsson, S. \& Heyman, B. Efficient IgG-mediated suppression of primary antibody responses in Fcgamma receptordeficient mice. Proceedings of the National Academy of Sciences of the United States of America 96, 2244-2249 (1999).

46. Zhang, Y. et al. Germinal center B cells govern their own fate via antibody feedback. $J$ Exp Med 210, 457-464 (2013).

47. Regules, J.A. et al. Fractional Third and Fourth Dose of RTS,S/AS01 Malaria Candidate Vaccine: A Phase 2a Controlled Human Malaria Parasite Infection and Immunogenicity Study. The Journal of infectious diseases 214, 762-771 (2016).

48. Koutsakos, M. et al. Circulating TFH cells, serological memory, and tissue compartmentalization shape human influenza-specific B cell immunity. Sci Transl Med 10 (2018).

49. Andrews, S.F. et al. High preexisting serological antibody levels correlate with diversification of the influenza vaccine response. J Virol 89, 3308-3317 (2015).

50. Bar, K.J. et al. Effect of HIV Antibody VRC01 on Viral Rebound after Treatment Interruption. The New England journal of medicine 375, 2037-2050 (2016).

51. Bar-On, Y. et al. Safety and antiviral activity of combination HIV-1 broadly neutralizing antibodies in viremic individuals. Nature medicine 24, 1701-1707 (2018). 
995 52. Mendoza, P. et al. Combination therapy with anti-HIV-1 antibodies maintains viral suppression. Nature 561, 479-484 (2018).

53. Escolano, A., Dosenovic, P. \& Nussenzweig, M.C. Progress toward active or passive HIV-1 vaccination. J Exp Med 214, 3-16 (2017).

54. Taylor, J.J. et al. Deletion and anergy of polyclonal B cells specific for ubiquitous membrane-bound self-antigen. J Exp Med 209, 2065-2077 (2012).

55. Kepler, T.B. Reconstructing a B-cell clonal lineage. I. Statistical inference of unobserved ancestors. F1000Res 2, 103 (2013).

56. Lefranc, M.P. et al. IMGT, the international ImMunoGeneTics information system. Nucleic Acids Res 37, D1006-1012 (2009).

57. $\mathrm{Wu}, \mathrm{X}$. et al. Rational design of envelope identifies broadly neutralizing human monoclonal antibodies to HIV-1. Science 329, 856-861 (2010).

58. Zavala, F., Cochrane, A.H., Nardin, E.H., Nussenzweig, R.S. \& Nussenzweig, V. Circumsporozoite proteins of malaria parasites contain a single immunodominant region with two or more identical epitopes. J Exp Med 157, 1947-1957 (1983).

59. Rodriguez, C.I. et al. High-efficiency deleter mice show that FLPe is an alternative to Cre-loxP. Nat Genet 25, 139-140 (2000).

60. Graewe, S., Retzlaff, S., Struck, N., Janse, C.J. \& Heussler, V.T. Going live: a comparative analysis of the suitability of the RFP derivatives RedStar, mCherry and tdTomato for intravital and in vitro live imaging of Plasmodium parasites. Biotechnol J 4, 895-902 (2009).

61. Rizzetto, S. et al. B-cell receptor reconstruction from single-cell RNA-seq with VDJPuzzle. Bioinformatics 34, 2846-2847 (2018). 


\section{Figure 1}
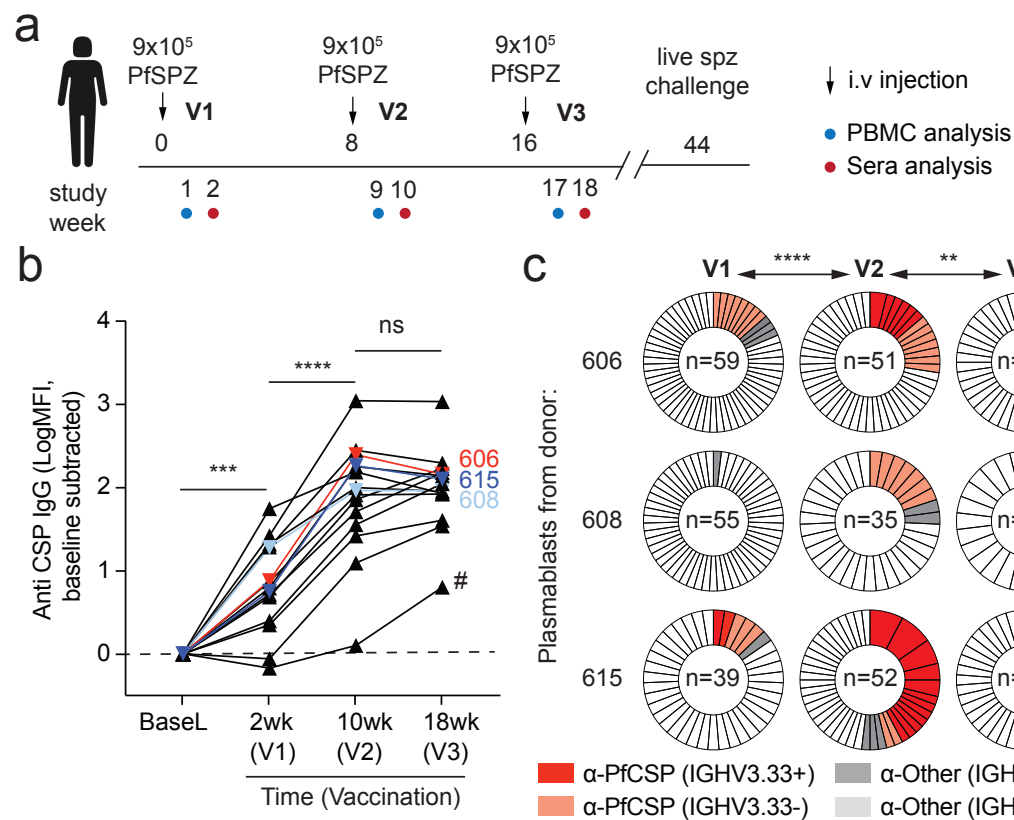

e
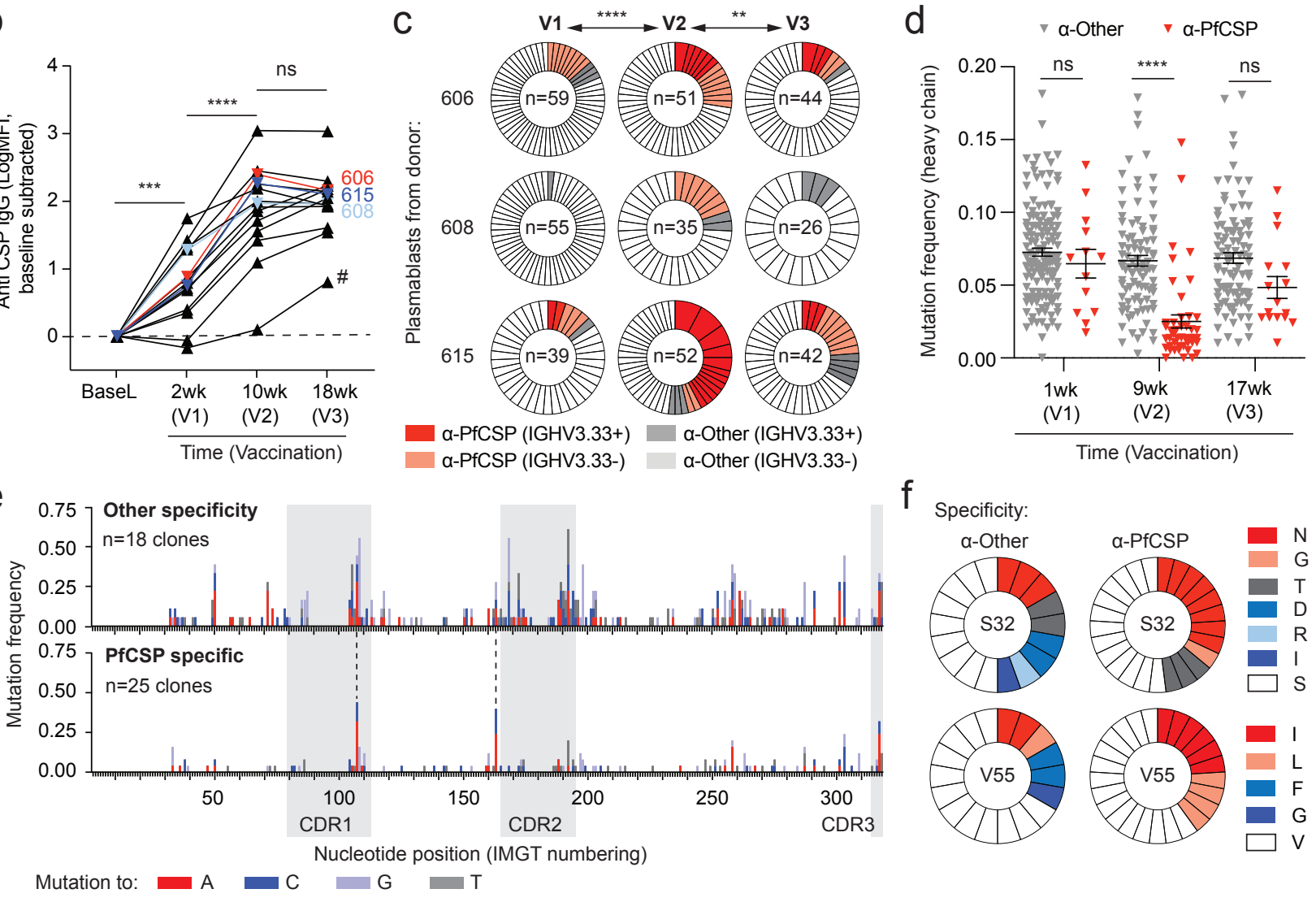

Figure 1: Limited memory B cell responses to PfCSP following repeated vaccination.

A. Schematic of the vaccination protocol for the VRC314 clinical trial of PfSPZ with the timing of sera and PBMC collection. B. Antibody responses to whole PfCSP measured by electrochemiluminescence at the stated timepoints after each immunization, antibody responses in individuals selected for downstream PB analysis are highlighted in colour; analysis was performed by repeated measured one-way ANOVA with Tukey's multiple comparisons test. C. Proportion of PfCSP specific PBs isolated from 3 donors after each immunization segregated by the use of the IGHV3.33 allele; each individual wedge indicates a unique clone. Analysis was performed by chi-squared test with subject as a blocking factor. D. Mutational frequency in the heavy chain gene of non-PfCSP specific PBs ( $\alpha$-other; grey circles) and PfCSP specific PBs ( $\alpha$-PfCSP; red triangles) after each immunization, bars show mean \pm s.d.; analysis by two-way ANOVA with subject as a blocking factor. E. Manhattan plots showing the location and frequency of mutations in the IGHV3.33 genes of non-PfCSP specific PB clones and PfCSP-specific PB clones pooled from the three subjects sequenced. F. Frequency of different amino acid changes at positions 32 and 55 in PfCSP and non-PfCSP specific PB clones. 
bioRxiv preprint doi: https://doi.org/10.1101/808543; this version posted October 18,2019 . The copyright holder for this preprint (which was not certified by peer review) is the author/funder, who has granted bioRxiv a license to display the preprint in perpetuity. It is made available under aCC-BY-NC-ND 4.0 International license.

Figure 2

a
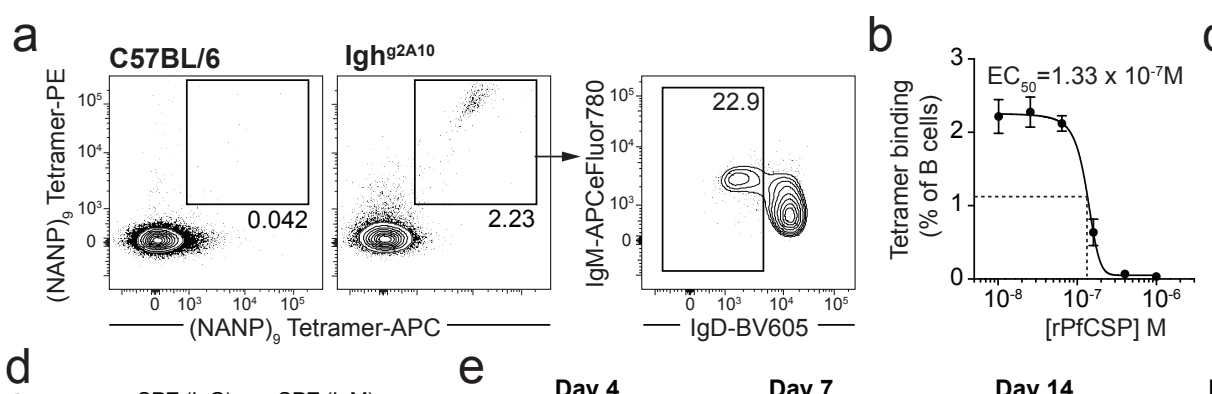

C $2 \times 10^{4} \mathrm{CD} 45.1^{+}$ $\operatorname{lgh}^{92 A 10} B$ cells
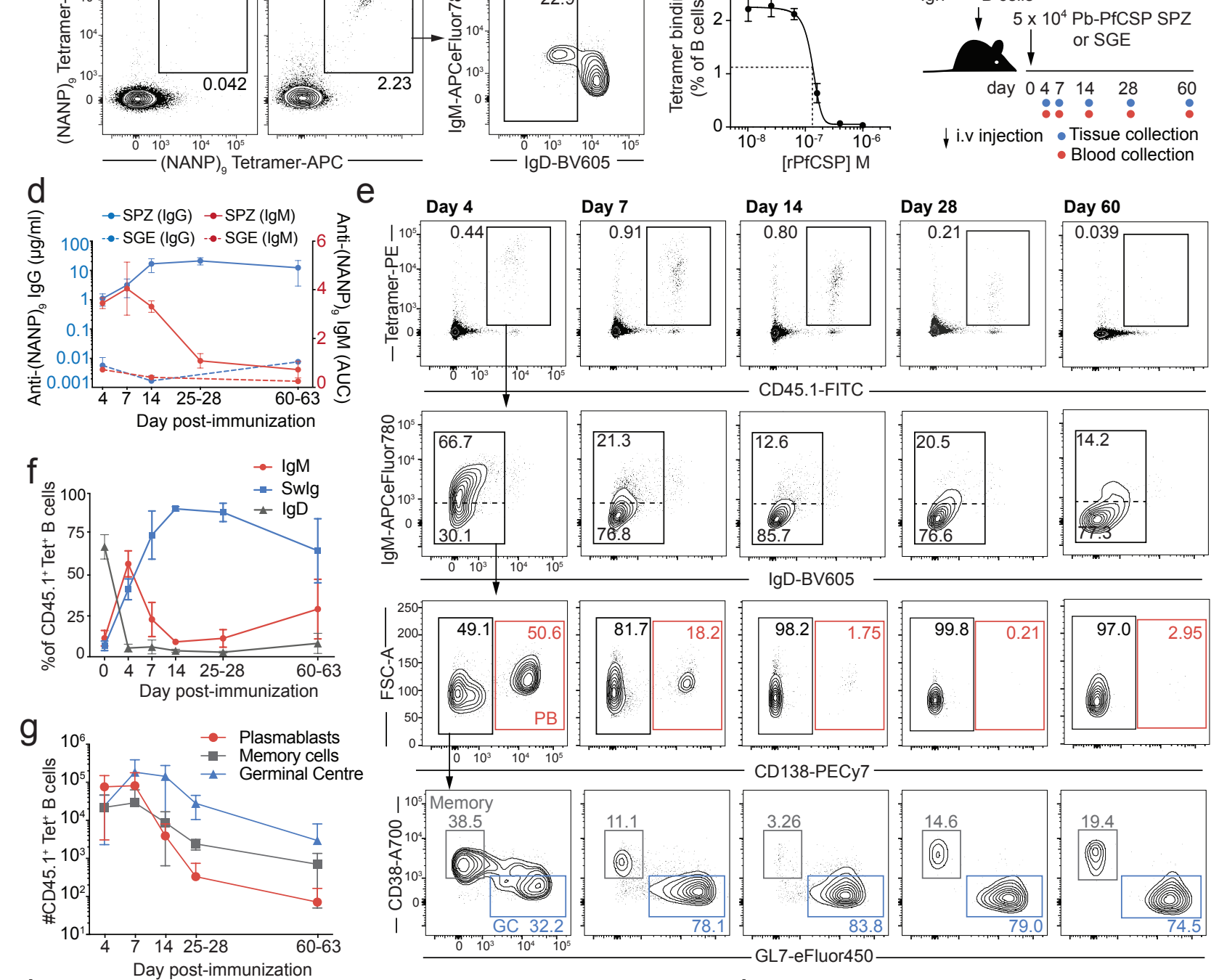

Day 28

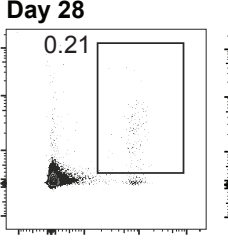

Day 60
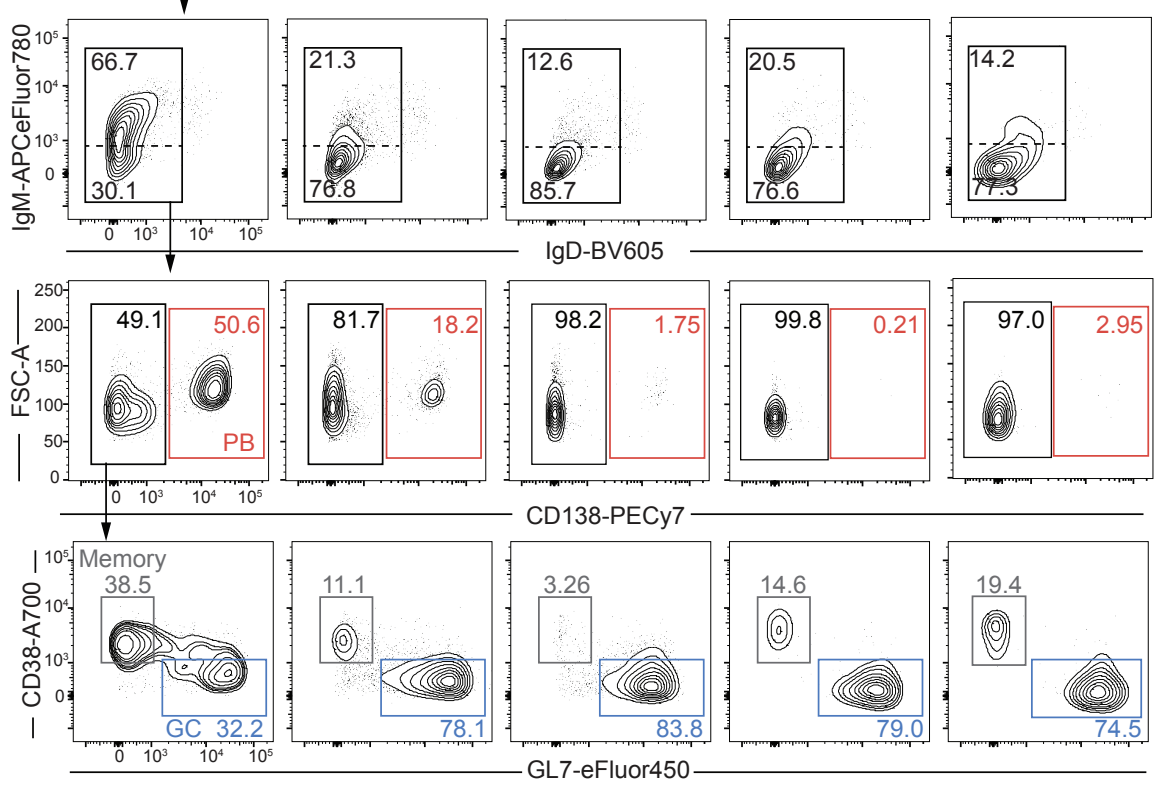

$\mathrm{h}$
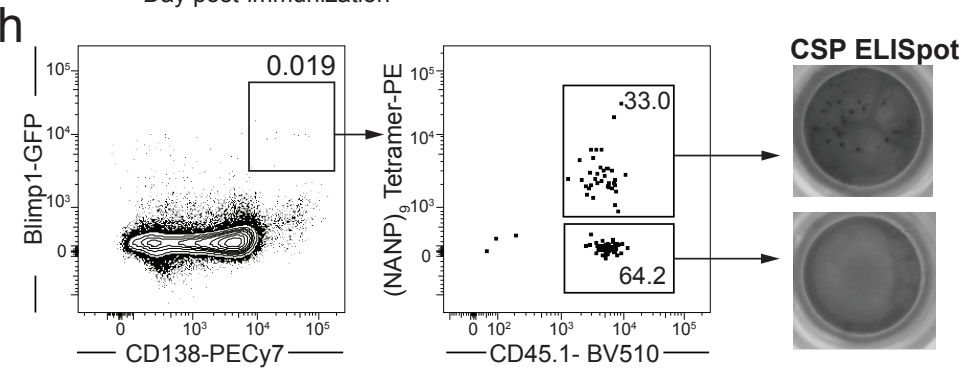

GL7-eFluor450

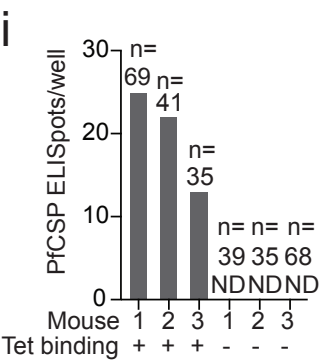

Figure 2: Development of Igh ${ }^{2 \mathrm{~A} 10}$ mice to track B cell responses to PfCSP.

A. Representative FACs plots (gated on B cells) showing the percentage of B cells that bind to (NANP) $)_{9}$-tetramers in C57BL/6 and lgh ${ }^{22 A 10}$ mice, and the $\operatorname{lgM}$ and $\operatorname{lgD}$ expression on these cells. $B$. Titration of the concentration of PfCSP required to block tetramer staining of $\operatorname{lgh}^{92 \mathrm{~A} 10}$ cells; $\operatorname{lgh}^{92 A 10}$ cells were incubated with the indicated concentrations of PfCSP, prior to (NANP) ${ }_{9}$-Tetramer staining and flow cytometry, analysis by non-linear regression. C. Schematic of immunization experiment in which mice received Igh $^{92 A 10}$ cells and were immunized with Pb-PfCSP SPZ (SPZ) or salivary gland extract (SGE). D. Concentration of anti-(NANP) $)_{n}$ antibodies in mice immunized 
as in C, left axis represents lgG (expressed as $\mu \mathrm{g} / \mathrm{ml}$ ) and right axis represents lgM expressed as area under the curve (AUC), means \pm s.d. shown. E. Representative flow cytometry plots showing the phenotypes of Igh ${ }^{92 A 10} \mathrm{~B}$ cells in mice immunized with Pb-PfCSP SPZ as outlined in C. F. Summary data showing the proportions of $\operatorname{lgh}^{92 \mathrm{~A} 10}$ cells that expressed $\operatorname{lgD}$, IgM or neither $(\mathrm{Swlg})$ at the indicated timepoints; means \pm s.d. shown. G. Summary data showing the proportions of Igh'2A10 cells that are PC, GC B cells or memory B cells at the indicated timepoints; means \pm s.d. shown. $\mathrm{H}$. Gating strategy for the identification and sorting of Igh ${ }^{2 \mathrm{~A} 10}$ BMPCs and representative PfCSP coated ELISpot wells probed with anti-IgG-HRP from mice immunized as in C. I. Summary data based on $\mathrm{H}$. showing the number of CSP-specific Igh" ${ }^{92 A 10}$ antibody secreting cells per leg from 3 mice as identified by ELISpot, the $n$ given above is the total number of cells in each gate that were sorted into each well. 
Figure 3

a

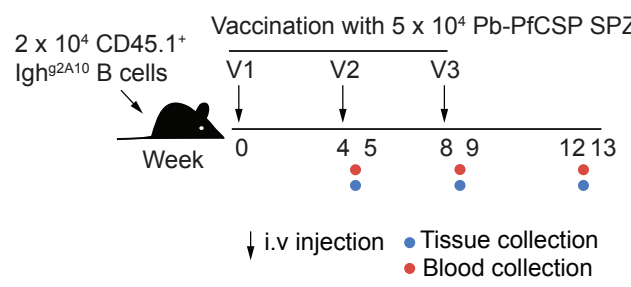

C $\quad$ Gated on Tet ${ }^{+} \mathrm{CD} 45.1^{+}$

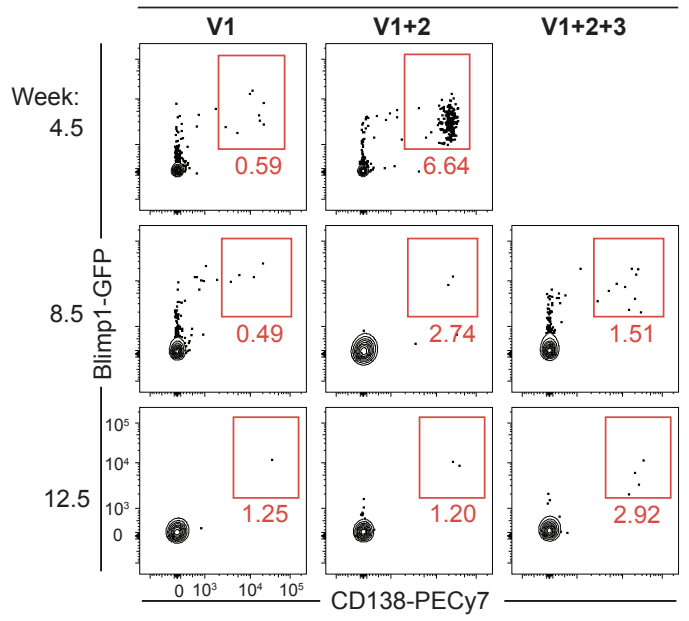

e

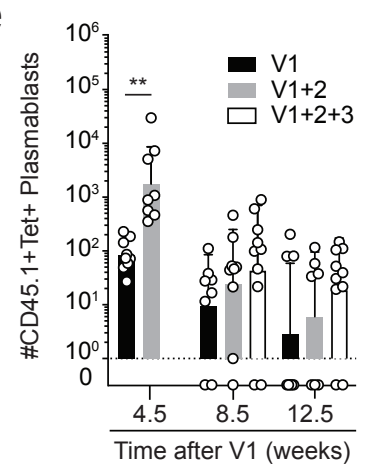

f

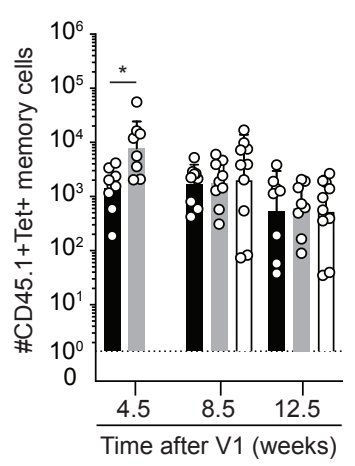

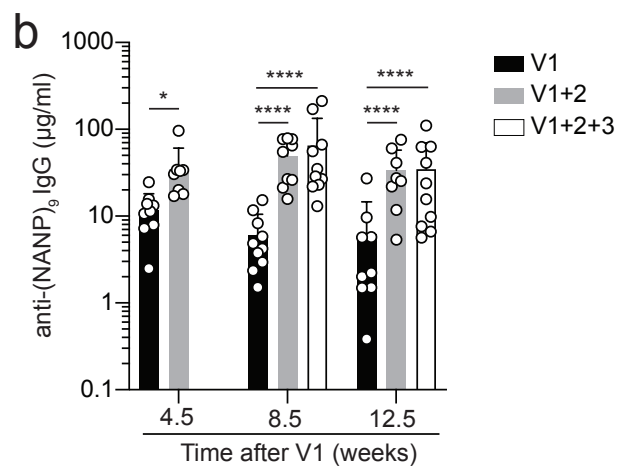

d

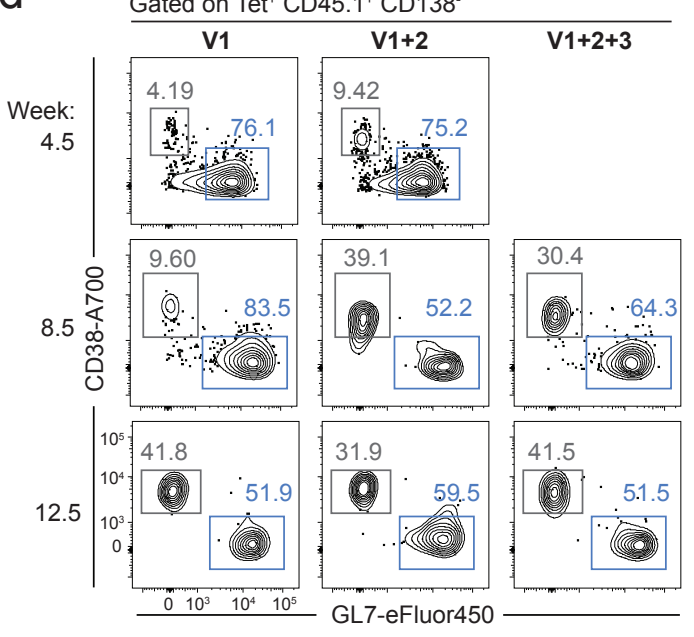

g

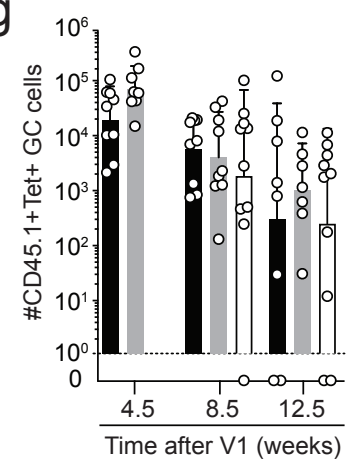

$\mathrm{h}$

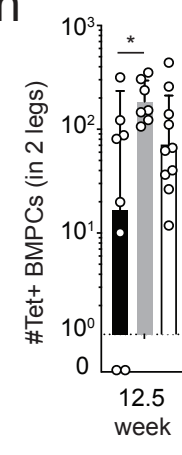

Figure 3: Limited memory B cell recall responses to PfCSP following repeated vaccination in mice.

A. Immunization schedule for the experiment: mice received $2 \times 10^{4}$ congenically marked Igha2A10 Blimp ${ }^{\text {gfp/t }}$ cells and were immunized 1, 2 or 3 times with $5 \times 10^{4} \mathrm{~Pb}$-PfCSP SPZ at 4 week intervals. 5 days after each boost and 33 days after the final boost blood, splenocytes and bone marrow was collected from the mice for analysis by ELISA and flow cytometry. B. Concentrations of anti-(NANP) $)_{\mathrm{n}}$ IgG in the sera at the indicated timepoints. C. Representative flow cytometry plots for the identification of Igh ${ }^{2 \mathrm{~A} 10} \mathrm{PBs}$ and plasma cells in the spleen. D. Representative flow cytometry plots for the identification of Igh ${ }^{\mathrm{g} 2 \mathrm{~A} 10} \mathrm{GC}$ B cells and memory cells in the spleen. Summary data for the analysis of spleen PBs (E), spleen memory B cells (F), spleen GC B cells (G) and BMPCs $(\mathrm{H})$ at the indicated timepoints; data are pooled from 3 replicate experiments, analysis was by 2-way ANOVA including the experiment as a blocking factor, bars represent means \pm s.d.. 


\section{Figure 4}

a

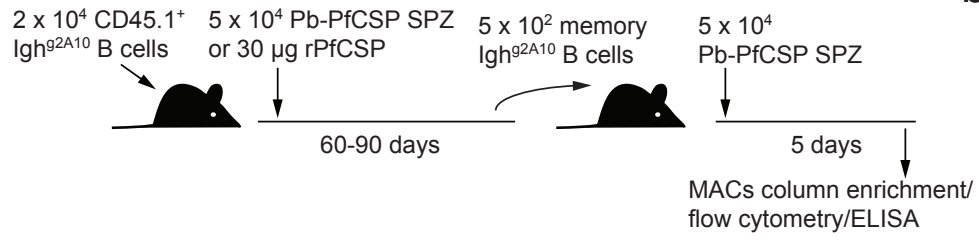

C

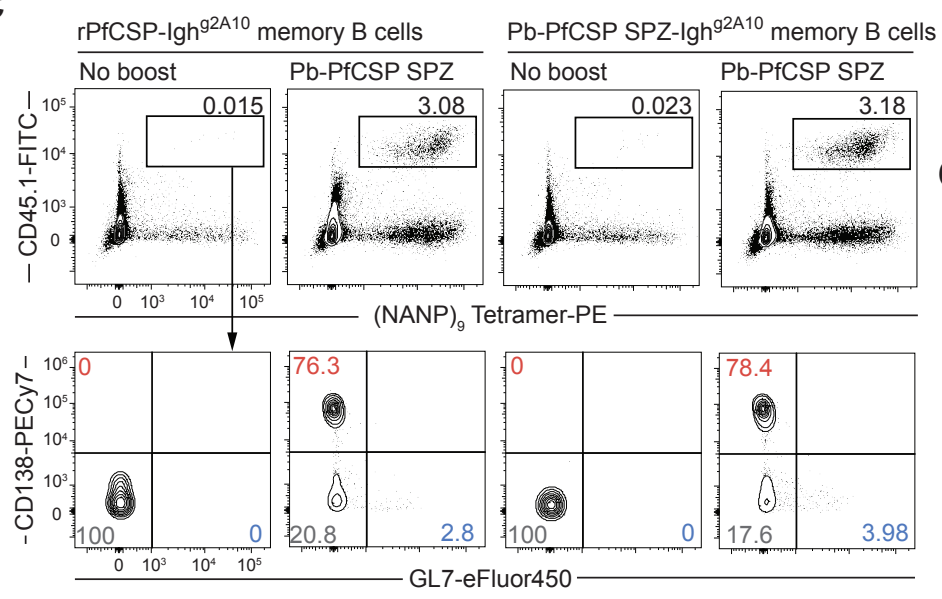

b


$\operatorname{lgh}^{\mathrm{g} 2 \mathrm{~A} 10}$ memory $\mathrm{B}$ cells

d $10^{5} \underbrace{*}_{* * * *} \quad$ e

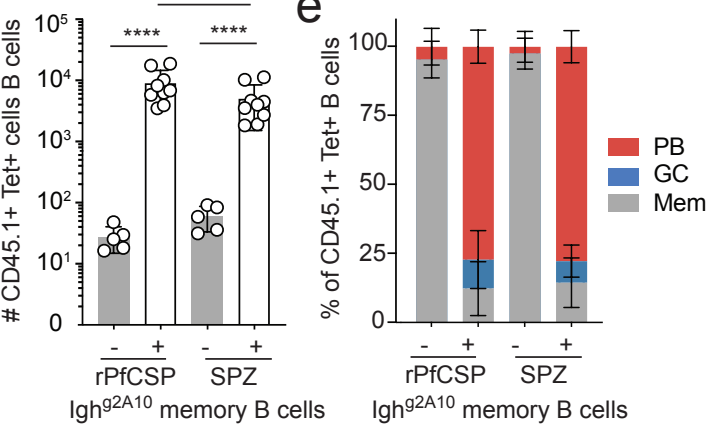

Figure 4: Memory B cells induced by sporozoite immunization are able to mount recall responses.

A. Schematic of the experiment showing the protocol for the generation and transfer of Igha2A10 memory B cells to naïve recipient mice and subsequent boosting with Pb-PfCSP SPZ. B. Levels (NANP) $)_{n}$-specific $\operatorname{lgM}$, and IgG in mice that received $\operatorname{lgh}^{92 \mathrm{A10}}$ memory $B$ cells 5 days after boosting with $5 \times 10^{4} \mathrm{~Pb}$-PfCSP SPZ compared to naïve mice that did not receive memory cells, but were immunized concurrently with $5 \times 10^{4} \mathrm{~Pb}-\mathrm{PfCSP} \mathrm{SPZ}$; data from a single experiment, bars show mean \pm s.d., analysis by one-way ANOVA with Tukey's multiple comparisons test. C. Representative flow cytometry plots of Igh ${ }^{92 A 10}$ memory cells recovered by magnetic bead purification from recipient mice immunized as in A. gated on $\mathrm{CD} 19^{+}$or $\mathrm{CD} 138^{+} \mathrm{B}$ cells and PBs. D. Quantification of the numbers of recovered cells in each group; bars show mean \pm s.d., data pooled from 2 experiments with analysis by two-way ANOVA with experiment as a blocking factor. E. Proportions of memory cells from (D) that had differentiated into PBs, GC B cells or retained a memory phenotype (Mem); means \pm s.d. shown. 
bioRxiv preprint doi: https://doi.org/10.1101/808543; this version posted October 18,2019 . The copyright holder for this preprint (which was not certified by peer review) is the author/funder, who has granted bioRxiv a license to display the preprint in perpetuity. It is made available under aCC-BY-NC-ND 4.0 International license.

\section{Figure 5}

a

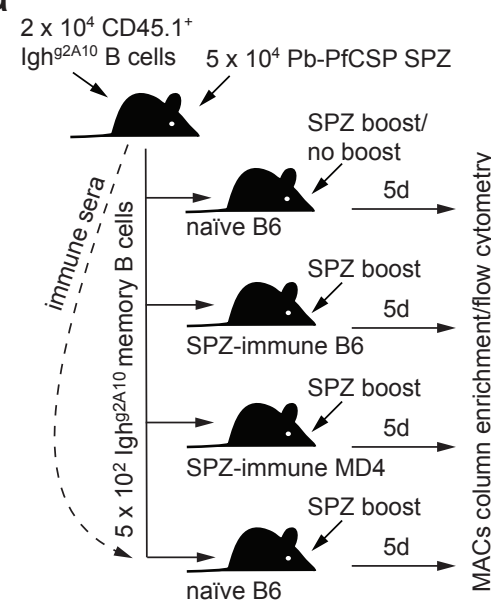

C

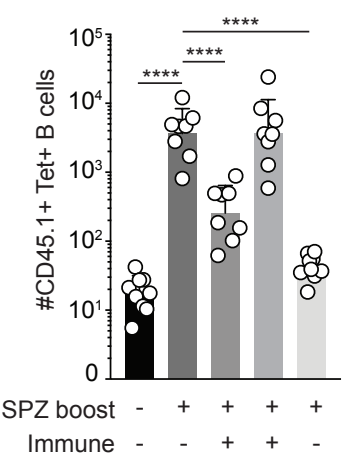

Background B6 B6 B6 MD4 B6

Immune Sera - - $\quad-\quad+$

$\mathrm{d}$ b

\begin{tabular}{|c|c|c|c|c|}
\hline \multirow{2}{*}{$\frac{\text { No boost }}{\text { naïve }}$} & \multicolumn{4}{|c|}{ Pb-PfCSP SPZ boost } \\
\hline & $\begin{array}{l}\text { naïve } \\
\text { (C57BL/6) }\end{array}$ & $\begin{array}{l}\text { SPZ-immune } \\
\text { (C57BL/6) }\end{array}$ & $\begin{array}{l}\text { SPZ immune } \\
\text { (MD4) }\end{array}$ & $\begin{array}{l}\text { naïve (C57BL/6)+ } \\
\text { immune sera }\end{array}$ \\
\hline
\end{tabular}
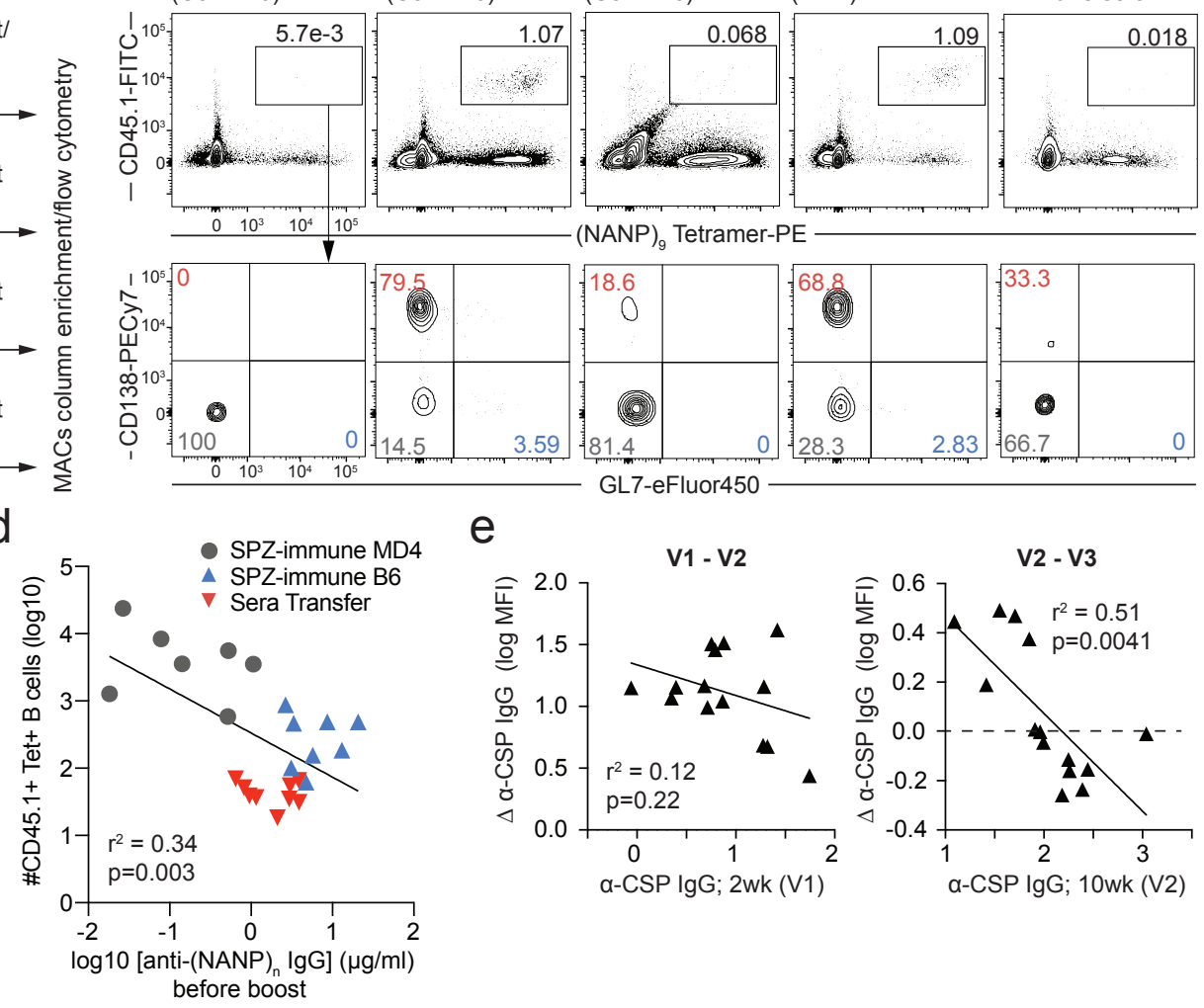

e
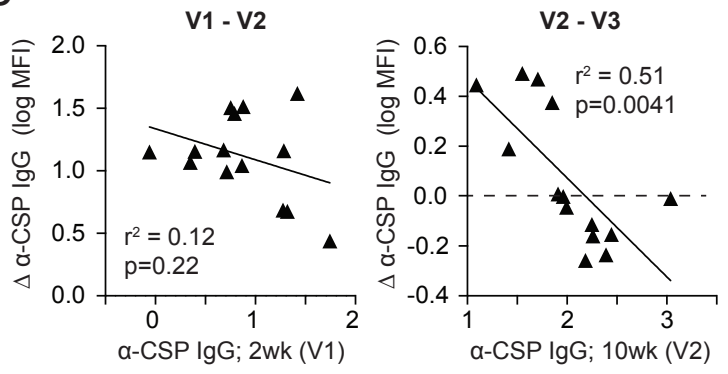

Figure 5: Antibody feedback regulates memory B cell responses in mice and humans.

A. Schematic of the experiment showing the generation of and transfer of memory cells and sera to different groups of naive and immune recipients prior to boosting with $5 \times 10^{4} \mathrm{~Pb}-\mathrm{PfCSP}$ SPZ. B. Representative flow cytometry plots of Igh ${ }^{2 \mathrm{~A} 10}$ memory cells recovered by magnetic bead purification from recipient mice immunized as in A. gated on CD19+ or CD138+ B cells and PBs. C. Quantification of the numbers of recovered cells in each group; data pooled from 2 experiments, bars show mean \pm s.d., with analysis by one-way ANOVA using Tukey's multiple comparisons test, with experiment as a blocking factor, only significant comparisons with the positive control group (Pb-PfCSP boosted naïve C57BL/6 recipients) are shown. D. Correlation of the sera titers of anti-(NANP) $)_{n}$ antibodies prior to boosting in the different groups of mice in A. with the subsequent size of the expansion of the CD45.1 $1^{+}$Tetramer $^{+}$B cell population after boosting; data pooled from 2 experiments, analysis by linear regression. E. Correlation of the response (change in anti-PfCSP antibody level) to V2 and V3 boosts with the titer of antibodies prior to the corresponding boost among the PfSPZ vaccinated individuals described in Figure 1A; analysis by linear regression. 
Figure 6
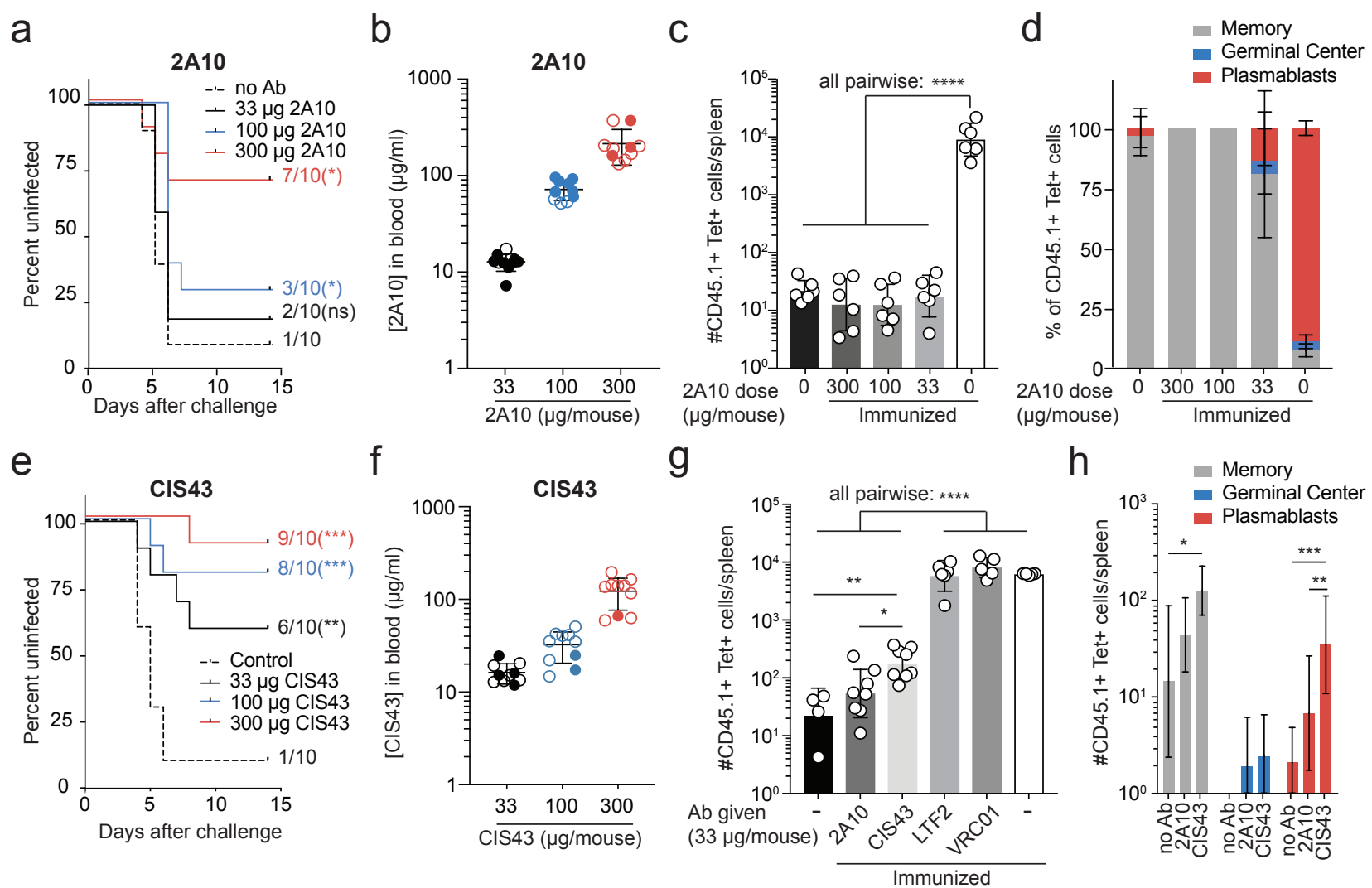

Figure 6: Sub-protective levels of antibody potently inhibit memory B cell responses.

A. Survival plots showing the proportion of uninfected mice after IV transfer of the specified amounts of 2A10 antibody and feeding by 7 infected mosquitoes; data pooled from 2 experiments, analysis by Log-rank (Mantel-Cox) test showing pairwise comparisons with the no antibody group. B. Concentration of 2A10 antibody in the blood 2 hours post-transfer via ELISA; hollow circles indicate protected mice, means \pm s.d. shown. C. Expansion of Igh ${ }^{2 A 10}$ memory $B$ cells (generated using Pb-PfCSP SPZ immunization and boosted as in figure 4A) in recipient mice that received the specified doses of 2A10; data pooled from 2 independent experiments and analysed by one-way ANOVA using Tukey's multiple comparisons test with experiment as a blocking factor. D. Proportions of Igh ${ }^{92 A 10}$ memory cells from (C) that had differentiated into PBs, GC B cells, or retained a memory phenotype. E. Survival plots showing the proportion of uninfected mice after IV transfer of the specified amounts of CIS43 antibody and feeding by 7 infected mosquitoes; data pooled from 2 experiments, analysis by Log-rank (Mantel-Cox) test showing pairwise comparisons with the no antibody group. F. Concentration of CIS43 antibody in the blood 2 hours post-transfer via ELISA; hollow circles indicate protected mice, means \pm s.d. shown. G. Expansion of memory $\mathrm{B}$ cells (generated using Pb-PfCSP immunization and boosted as in figure $4 \mathrm{~A}$ ) in recipient mice that received $33 \mu \mathrm{g}$ of the specified anti-CSP (2A10 and CIS43) antibodies, and antibodies of irrelevant specificity (LTF2 and VRC01); data pooled from 2 independent experiments and analysed by one-way ANOVA using Tukey's multiple comparisons test with experiment as a blocking factor, means \pm s.d. shown. $H$. Numbers of recovered PB, GC and memory CD45.1+ Tetramer+ cells from the no antibody/no immunization, 2A10 and CIS43 groups in G; means \pm s.d. shown; analysis by two-way ANOVA with experiment as a blocking factor. 


\section{Figure 7}

a

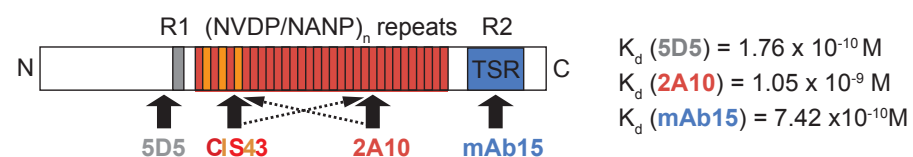

b

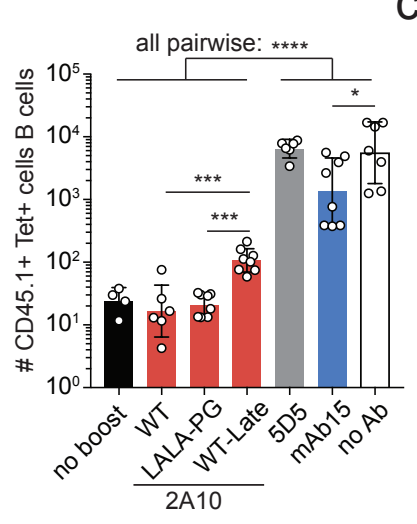

C

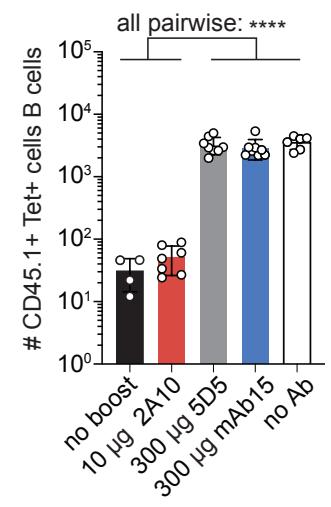

d

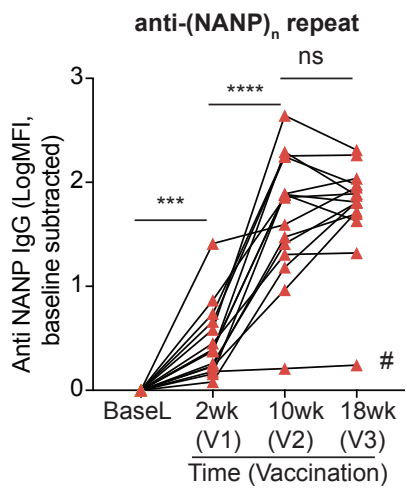

anti-C terminal domain

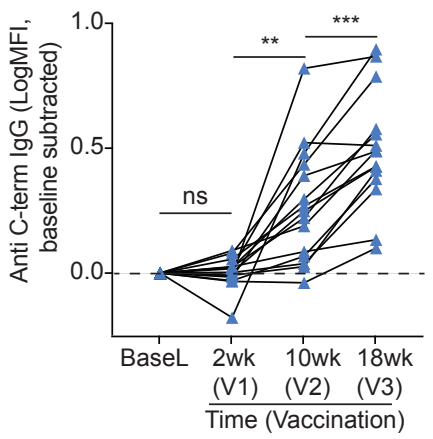

e
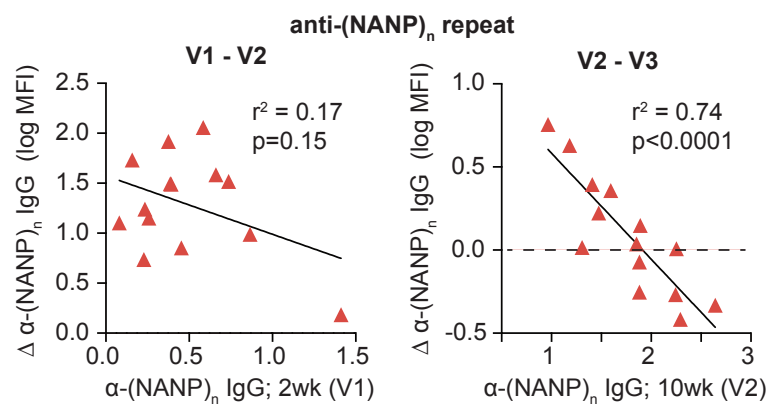

f

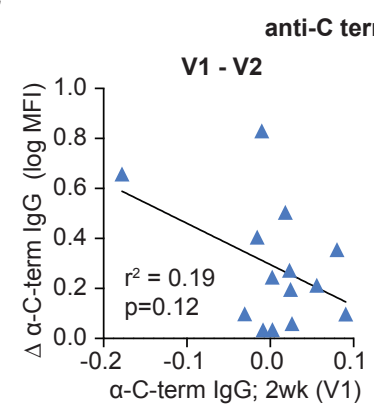

Figure 7: Antibody feedback occurs via epitope masking and allows the diversification of the antibody response.

A. Schematic of the CSP molecule showing the binding sites and dissociation constants of the different antibodies used in this study. B. Expansion of memory B cells in the presence of antibodies (30 $\mathrm{gg} / \mathrm{mouse}$ ) targeting non-repeat regions of CSP (5D5 and mAb15), anti-repeat Fc-dead 2A10 (2A10-LALA-PG) and 2A10 transferred 4 hours post sporozoite delivery; memory cells were generated via Pb-PfCSP SPZ immunization and transferred as in figure 4A and expansion measured 5 days post boosting with $5 \times 10^{4} \mathrm{~Pb}$-PfCSP SPZ. C. Expansion of memory $\mathrm{B}$ cells in the presence of anti-PfCSP antibodies at different concentrations; memory cells were generated via $\mathrm{Pb}-\mathrm{PfCSP}$ SPZ immunization and transferred as in figure 4A and expansion measured 5 days post boosting with $5 \times 10^{4} \mathrm{~Pb}$-PfCSP SPZ. D. Antibody responses specific for the (NANP) $)_{n}$-repeat and C-terminal domain of CSP in PfSPZ vaccinated subjects (described in Figure 1A) analysis was performed by repeated measures one-way ANOVA with Tukey's multiple comparisons test; \# indicates one individual who did not respond and was excluded from subsequent analysis. F.

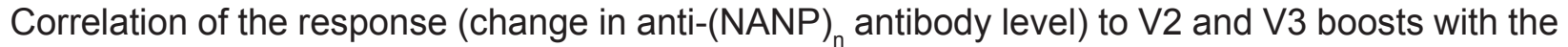
titer of antibodies prior to the corresponding boost; analysis by linear regression. G. Correlation of the response (change in anti-terminal antibody level) to V2 and V3 boosts with the titer of antibodies prior to the corresponding boost; analysis by linear regression. 